\title{
Coherence Resonance Behavior of FitzHugh-Nagumo Neurons Induced by Electromagnetic Field Driven by Phase Noise
}

\author{
Kaijun Wu, Huan Zheng, and Tao Li iD \\ School of Electronics and Information Engineering, Lanzhou Jiaotong University, Lanzhou 730070, China \\ Correspondence should be addressed to Tao Li; 0619679@stu.lzjtu.edu.cn
}

Received 15 September 2021; Revised 30 December 2021; Accepted 31 December 2021; Published 27 January 2022

Academic Editor: Ya Jia

Copyright (c) 2022 Kaijun Wu et al. This is an open access article distributed under the Creative Commons Attribution License, which permits unrestricted use, distribution, and reproduction in any medium, provided the original work is properly cited.

\begin{abstract}
Noise exists widely in the nervous system, and plays a crucial role in the nervous system information processing. Noise can not only enhance but also weaken the ability of the nervous system to process information. Neurons are in a complex and variable electromagnetic field. Electromagnetic induction plays an important role in regulating the changes of neuronal membrane potential. Therefore, this paper simulates the electromagnetic field environment of the nervous system with a memristor and analyses the rich coherence resonance behavior of FitzHugh-Nagumo (FHN) neuron system under the drive of phase noise. By taking the amplitude, period and noise intensity of phase noise as the main parameters and the parameters of memristor as auxiliary parameters, the two-parameter changes are made from the angle of the amplitude and period of phase noise, the amplitude and intensity of phase noise, and the noise intensity and period of phase noise, respectively. The dynamic behaviors of coherent resonance of FHN neuron system are analyzed from the amplitude and period, amplitude and intensity as well as intensity and period of phase noise, respectively. When the amplitude and period of the phase noise and the intensity and period of the phase noise are used as independent variables for the two-parameter analysis, the FHN neuron system shows rich dynamic behaviors such as coherence mono-resonance, coherence bi-resonance and coherence multi-resonance. Especially when the amplitude and period of phase noise change as two-parameter, the system presents a coherence resonance of discharge pattern with period-adding cluster discharge at the valley. When the amplitude and intensity of phase noise are taken as independent variables for two-parameter analysis, FHN neuronal system presents single or dual coherence resonance at any value of noise intensity with the change of phase noise amplitude. The simulated results show that the FHN neuron system demonstrates rich coherence resonance behaviors under the drive of phase noise when the effect of electromagnetic induction in the nervous system is simulated by memristor.
\end{abstract}

\section{Introduction}

The nervous system is a complex network of huge numbers of neuron cells. Neurons are the basic unit of the nervous system whose dynamic characteristics play an important role in the operation of the neuron system. The discharging modes of neuronal systems exhibit complex and contradictory nonlinear dynamic phenomena such as bifurcation, chaos, stochastic resonance, coherence resonance, and vibrational resonance [1-7]. Noise exists widely in the natural system, therefore, as a huge neural network, the existence of noise is inevitable. The existence of noise in the nervous system may cause the system to behave in ways that defy common sense. Generally speaking, as a kind of system interference factor, noise breaks the original stability of the system, thus bringing adverse and harmful effects to the system. However, studies at home and abroad show that the introduction of noise in nonlinear systems improves the information processing ability of the nervous system and makes the system more stable [8-13].

Phase noise is a typical bounded noise which causes the random fluctuation of the phase of the output signal. The nervous system is an extremely complex environment, and it bears complex and variable random external input sources. Therefore, the study of phase noise in nervous system is significant. In recent years, experiments with sensory and biological systems have demonstrated the existence of phase noise [14-16]. In addition, phase noise is widely used to 
describe phase fluctuations in oscillators and circuits [17]. Therefore, it is of great value to introduce phase noise into the nervous system to study the coherence resonance behavior of the nervous system under the action of random forces. Considering the distance difference between sensory neuron and signal source, the signal received by neuron sensor has phase shift characteristic. In 1993, Hu et al. found that in the absence of external weak periodic signal input, the output of nonlinear system becomes ordered and exhibits the behavior similar to stochastic resonance, which is known as "uniform resonance", "coherence resonance" (CR) or "autonomous stochastic resonance" (SR) [18]. Since coherence resonance is closer to the natural environment than stochastic resonance, so it has wider research significance and has received more attention from scholars. Coherence resonance refers to a phenomenon whereby addition of certain amount of noise in excitable system makes its oscillatory responses most coherent. Thus a coherence measure of stochastic oscillations attains an extremum at optimal noise intensity, hence the word "resonance" $[19,20]$. In recent years, the research on coherence resonance behavior of nervous system has become a hot issue, and a large number of research results have been published. Ditzinger et al. studied the response of two autonomous nonlinear systems, one bistable and the other monostable, to additive white noise in certain parameter regions numerically. They show that the additive white noise can induce coherent oscillations, and both autonomous systems show optimum responses, represented by the maximum signal-to-noise ratio under certain noise intensity. This phenomenon is thus termed as stochastic resonance without external periodic force [21]. Liu et al. studied the effects of phase noise and autosynapses on the resonance dynamics of improved Fitzhugh-Nagumo (FHN) neurons in the electromagnetic induction environment. It is found that phase noise can cause double coherence resonance, and if the period and feedback gain of phase noise are changed separately, the behavior similar to resonance will also occur [22]. Ding et al. studied the improvement of coherence resonance degree caused by inhibitory self-feedback and the potential post inhibitory rebound (PIR) mechanism in the HodgkinHuxley $(\mathrm{HH})$ model extend the inhibitory self-excitation phenomenon to the stochastic system, and proposed potential measures to adjust the degree of coherence resonance and information processing [23]. Jia et al. studied the counterintuitive phenomenon of inhibitory autosynaptic enhancement of coherence resonance in $\mathrm{HH}$ neural networks, which was well explained by the peak of inhibition rebound (Pir). They also introduced the Gaussian white noise into the excitatory coupled $\mathrm{HH}$ neuron network, and studied the coherence resonance dynamic behavior of the system was studied in detail on a plane composed of every two parameters such as noise intensity, adaptive intensity and time delay [24]. Ryashko et al. studied the random Hindmarsh-Rose (HR) neuron model of the toroidal canal area in parameter space and demonstrated that noise can transform the toroidal rod body into a torus with large amplitude [25]. Wang et al. studied the coherence resonance behavior of a two-dimensional Rulkov neuron system with stable sub-thresholding properties and found that noise induced neurons to generate coherence bi-resonance [26]. Liu et al discovered the coherence resonance behavior of nonlinear neuron system under the action of color correlation noise, and mainly analyzed the influence of additive and multiplicative color noise on the coherence resonance of neuron system [27]. Jia et al. studied the coherence biresonance behavior of FHN neurons induced by phase noise, and explained that the reason for coherence bi-resonance is that the period of phase noise is much larger than the internal firing period of neurons $[28,29]$. José M. Casado. et al studied the coherence resonance behavior of the simplified FHN neuron model $[30,31]$.

In 1971, when studing the classical electromagnetic field theory, professor Cai Shaotang predicted the fourth largest physical and electronic component in addition to resistance, capacitance and inductance: memristor [32]. After that, when HP Lab studied the properties of titanium dioxide, it happened to find the existence of memristor [33], which triggered a wave of memristor research. Although the memristor itself has a nanoscale size, it has a very strong storage capacity, and is not easy to lose information when losing power [34-37], so it is applied in various fields. In the study of biological nervous system, the characteristics of memristors are very similar to those of synapses, so the research on memristor self-synapse and memristor coupling synchronization has become a hot spot. Zhang. et al designed the Josephson junction circuit with memristor, discussed the dynamic response and potential mechanism of behavior selection, and studied the chaotic encryption of the Josephson junction circuit with memristor coupling [38]. On the basis of HR neuron model, Ma et al. considered the effect of electromagnetic induction on the coupling phase synchronization of neurons and found that the external electric field could induce the phase synchronization of neurons [39]. Ge et al. used an improved FHN neuron model of electromagnetic induction to study the vibrational resonance of single FHN neuron and the vibrational resonance of feedback neural network system under different electromagnetic induction and pre-feedback [40]. Because of the extremely special nature of memristor, it is of great significance to introduce it into the nervous system.

$\mathrm{HH}$ neuron model was first proposed by Hodgkin and Huxley, and in recent years, the $\mathrm{HH}$ neuron model has been studied in detail from multiple dynamics respects such as bifurcation, synchronization and resonance. FitzHugh [41] and Nugumo [42] proposed a two-dimensional simplified FHN neuron model with rich dynamic characteristics $[43,44]$. This paper simulates the electromagnetic environment of the nervous system with a memristor and analyses the rich coherence resonance behavior of the FHN neuronal system driven by phase noise. In the second part, this paper mainly introduces the random FHN neuron system model, the value of system parameters, the type of random force and the measurement standard of coherence resonance. The third part mainly takes the amplitude, period and noise intensity of phase noise as the main change parameters, and takes the parameter change of the memristor as the auxiliary condition. And it analyzes the dynamic 
behavior of the coherent resonance of the FHN neuron system under the two-parameter variation from three perspectives: the amplitude and period of the phase noise, the amplitude and noise intensity of the phase noise, and the noise intensity and period of the phase noise. The fourth part summarizes the interesting coherence resonance behavior of FHN system with memristor regulator driven by phase noise.

\section{Model introduction}

To analyze the random dynamic behavior of a non-linear neuronal system under the influence of magnetic flux variation and phase noise, this paper introduces flux-controlled memristor and phase noise into FHN neuron model, and analyzes the coherence resonance behavior of FHN neuron system. The model is established as follows:

$$
\begin{aligned}
\varepsilon \frac{\mathrm{d} x}{\mathrm{~d} t} & =x-\frac{x^{3}}{3}-y+k \rho(\varphi) x, \\
\frac{\mathrm{d} y}{\mathrm{~d} t} & =x+a+B \sin (z(t)), \\
\frac{\mathrm{d} z}{\mathrm{~d} t} & =\frac{2 \pi}{T}+\xi(t), \\
\frac{\mathrm{d} \varphi}{\mathrm{d} t} & =k_{1} x-k_{2} \varphi .
\end{aligned}
$$

In Eq. (1)-(2), as the fast variable of the system, the membrane potential is represented by $x$; as the slow variable of the system, the recovery variable is represented by y . $x$ and $\mathrm{y}$ are distinguished by time ratio constant $\varepsilon$, and $\varepsilon \ll 1$. To ensure the rapid evolution of the membrane potential with the change of time, let $\varepsilon=0.01$. Parameter $a$ determines the dynamic behavior of the system. When $|a|>1$, FHN neurons show resting state or subthreshold oscillation state. When $|a|<1$, FHN neurons are in the nerve pulse discharge state $[28,29]$.

In (1), $\rho(\varphi)$ denotes memductance of flux-controlled memristor, and $\rho(\varphi)=\alpha+3 \beta \varphi^{2}$. Where in $k \rho(\varphi) x$ represents the feedback current of electromagnetic induction on the membrane potential, and regulates the excitability of the cell membrane potential. It is expressed as:

$$
\begin{aligned}
i^{\prime} & =\frac{\mathrm{d} q(\varphi)}{\mathrm{d} t}=\frac{\mathrm{d} q(\varphi)}{\mathrm{d} \varphi} \frac{\mathrm{d} \varphi}{\mathrm{d} t} \\
& =\rho(\varphi) x=k \rho(\varphi) x .
\end{aligned}
$$

In (2), $B \sin (z(t))$ represents the phase noise, $B$ and $\sin (z(t))$ represent the amplitude and phase of the phase noise respectively. Specifically, $z(t)=2 \pi t / T+W(t)$, wherein $T$ represents the period of phase noise, and $W(t)$ represents the Wiener process. Then the derivative of $z(t)$ is shown in (3).

In (3), $D$ represents the noise intensity of Gaussian white noise $\xi(t)$ which meets the following statistical properties, as shown in (6):

$$
\begin{aligned}
\langle\xi(t)\rangle & =0, \\
\left\langle\xi(t) \xi\left(t^{\prime}\right)\right\rangle & =2 D \delta\left(t-t^{\prime}\right) .
\end{aligned}
$$

In (4), $\varphi$ represents the magnetic flux across the membrane, $k_{1} x$ represents the magnetic induction change induced by the membrane potential, and $k_{2} \varphi$ represents the flux leakage.

\section{Numerical simulation}

In this system, in order to make the system in the state of subthreshold oscillation, make the value of system parameter $a$ near the Hopf bifurcation point. The amplitude $B$, period $T$ and noise intensity $D$ of phase noise are taken as the noise variables affecting the system, which are used to analyze the coherence resonance behavior of the system. And their measurement intervals are $B \in[0,0.8], T \in[0,40]$ and $D \in[0,0.8]$, respectively. The electromagnetic field feedback gain coefficient $k$, the memristor parameters $k_{1}$ and $k_{2}$ of the flux-controlled memristor are used to simulate the electromagnetic field environment in which the system is.

In order to quantitatively describe the degree of order of the neuron system under the action of random noise, we can measure the intensity of coherence resonance of the system by calculating the value of coherence resonance coefficient $R$, which is specifically defined as follows:

$$
\begin{aligned}
R & =\frac{\sqrt{\left\langle T_{0}^{2}\right\rangle-\left\langle T_{0}\right\rangle^{2}}}{\left\langle T_{0}\right\rangle}, \\
& =\frac{\sqrt{\operatorname{var}\left(T_{0}\right)}}{\left\langle T_{0}\right\rangle},
\end{aligned}
$$

Where in $T_{0}$ represents interspike intervals sequence of the system, $\left\langle T_{0}\right\rangle$ represents the mean value of interspike intervals sequence, $\operatorname{var}\left(T_{0}\right)$ represents the variance of the interspike intervals sequence, which represents the fluctuation degree of $T_{0}$. The smaller the coherence resonance coefficient $R$ is, the stronger the discharge regularity of the neuron system is. Otherwise, the weaker. In order to eliminate the interference of random results on the degree of system coherence resonance, take longer interspike intervals as possible. And average the results of the same group of parameters several times to ensure that the measurement standard reflects the system state more accurately.

This paper, analyzes the random dynamic behavior of FHN neurons by variable-step fourth-order Runge-Kutta algorithm, and the initial state of the system is made $(0.1,0.1$, $0.1,0.1)$. In order to eliminate the influence of the phase noise and the flux-controlled memristor, the amplitude of the phase noise is set as $B=0$ and the feedback adjustment parameter of the flux-controlled memristor is set as $k=0$. Figure 1 shows the bifurcation diagram of FHN neuron deterministic system under the change of parameter $a$. When $|a|>1$, FHN neurons shows resting state or subthreshold oscillation state; when $|a|<1$, FHN neurons were in the state of nerve pulse discharge. In order to study the stochastic dynamic behavior of FHN neurons driven by 


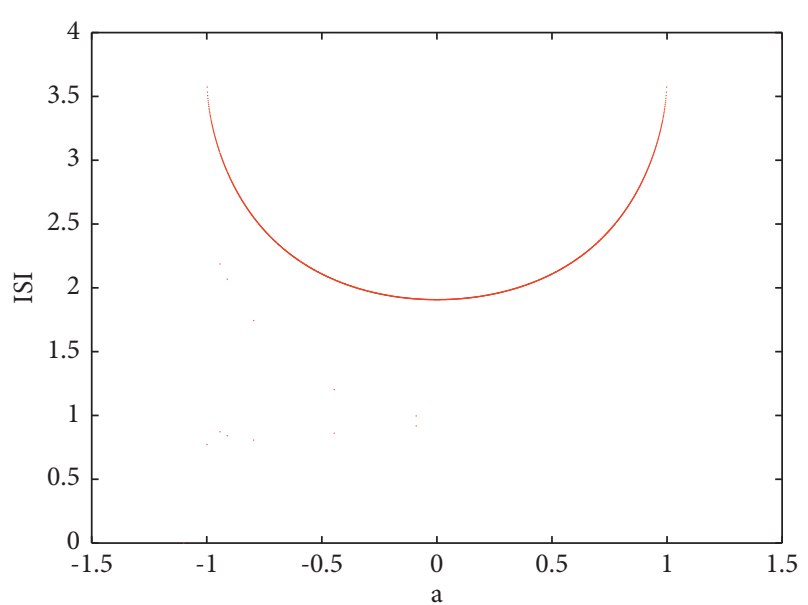

FIGURE 1: The bifurcation diagram of FHN neuron deterministic system with parameter $a$.

phase noise under the action of memristor, parameter $a$ was taken as the control parameter of the initial state of FHN neuron system discharge, and the value near Hopf bifurcation point was taken as the initial state of FHN neuron coherence resonance. Figure 2 shows the different discharging states of the FHN neuronal system when the control parameter $a$ takes the value before the bifurcation point, at the bifurcation point and after the bifurcation point respectively. Figure 2(a) shows when parameter $a=0.8$, FHN neurons show peak discharge rhythm of period 1 . Figure 2(b) shows when parameter $a=1.0$, FHN neurons show dense subthreshold oscillating discharge state. Figure 2(c) shows when parameter $a=1.0005$, compared with Figure 2(b), the amplitude of the subthreshold oscillation discharge of FHN neurons decreases gradually with the increase of parameter $a$. Figure 2(d) shows when parameter $a=1.05$, remove the initial unstable discharge state of the system, FHN neurons show a discharge state of a smooth line parallel to the time axis, namely the resting state. In this section, the value of system control parameter $a$ is set to 1.05 , that is, FHN neurons show a resting state, which is used to study the coherence resonance behavior of FHN neurons.

\subsection{Coherence Resonance Behavior of FHN Neurons with} Variations of Amplitude B and Period T of Phase Noise. To investigate the coherence resonance behavior of FHN neuronal systems when the phase noise amplitude $B$ and period $T$ take different values, this section sets the fluxcontrolled memristor parameters as $k=0.1, k_{1}=0.2$, $k_{2}=0.8, \alpha=0.4$ and $\beta=0.02$, and sets the noise intensity of phase noise $D=0.1$. By setting the phase noise amplitude $B \in[0,0.8]$ and the period value $T \in[0,40]$ in the FHN neuron system, it is found that the FHN neuron system exhibits coherence multi-resonance dynamic behavior. Figure 3 shows temporal evolution of membrane potential $x$ in FHN neuron system after the addition of flux-controlled memristor. During the evolution of membrane potential $x$, random spikes with minimal amplitudes appear, but the neuronal system is still in a resting state of discharge.
In Figure 4, when the phase noise amplitude $B \in[0,0.8]$ and the period value $T \in[0,40]$, the FHN neuron system shows coherence multi-resonance behavior. It is found in Figure 4(a) that when the phase noise amplitude $B$ is determined to be a certain value, the FHN neuron system exhibits the dynamic phenomenon of coherence multiresonance with the gradual increase of the period value $T$. It can be easily found from the figure that when phase noise period $T \in[0,6.5)$, with the gradual increase of phase noise period, FHN neuron system has changed from the first valley to the first peak, then from the first peak to the second valley, then from the second valley to the second peak, and then from the second peak to the third valley. That is, there is a coherence multi-resonance phenomenon in the system. In this process, the coherence resonance coefficient $R$ undergoes two valleys. When the coherence resonance coefficient $R$ is in the valley, $R$ obtains the minimum value in the local range, and then the discharge regularity of the system is the strongest. The optimal discharge state of FHN neuron system is found around phase noise period $T \in[0,6.5)$ and $T \in[6.5,40]$, and the degree of regularity is relatively optimal. When phase noise period $T \in[6.5,40]$, with the gradual increase of phase noise period $T$, the FHN neuron system experienced 7 peaks and 8 valleys successively, and showed concentric circle diffusion. By comparing the phase noise periods $T \in[0,6.5)$ and $T \in[6.5,40]$, it is found that values of coherence resonance coefficients at two valleys in the interval $T \in[0,6.5)$ are much smaller than those at eight valleys in the interval $T \in[6.5,40]$, And from the perspective of the interval $T \in[0,40]$ as a whole, the value of coherence resonance coefficient $R$ at the valley shows an overall upward trend, indicating that with the increase of the period value of phase noise, the degree of system regularity gradually deteriorates when coherence resonance occurs. It is observed in Figure 4(b) that coherence resonance occurs in the system within the interval $T \in[3,5.5), \quad T \in[5.5,10)$ and $T \in[13.5,16.5)$ of phase noise period as the phase noise amplitude $B$ changes. When $T \in[3,5.5)$, the system shows coherence mono-resonance, while when $T \in[5.5,10)$ or $T \in[13.5,16.5)$, the system shows more obvious coherence multi-resonance behavior, with the smallest coherence resonance coefficient value $R$ at the first valley. In conclusion, under the variation of phase noise amplitude $B$ and period $T$, FHN neurons show coherence multi-resonance behavior, in which the coherence resonance phenomenon is the most obvious and the system discharge is more orderly in the local region.

In order to verify the correctness of the above conclusions, set phase noise amplitude $B=0.76$ and phase noise period were selected as the system variation parameters to analyze the coherence multi-resonance behavior and the change of discharge regularity degree of FHN neuron system. Figure 5(a) shows the relationship between the coherence resonance coefficient $R$ and the period of phase noise $T$. When the period of phase noise $T \in[0,7)$, the coherence resonance coefficient $R$ experienced two valleys $T=3$ and $T=5.5$, that is, the two states with optimal neuronal membrane potential discharge regularity, which is shown in Figure 5(c) and (e). Figure 5(c) shows the most 


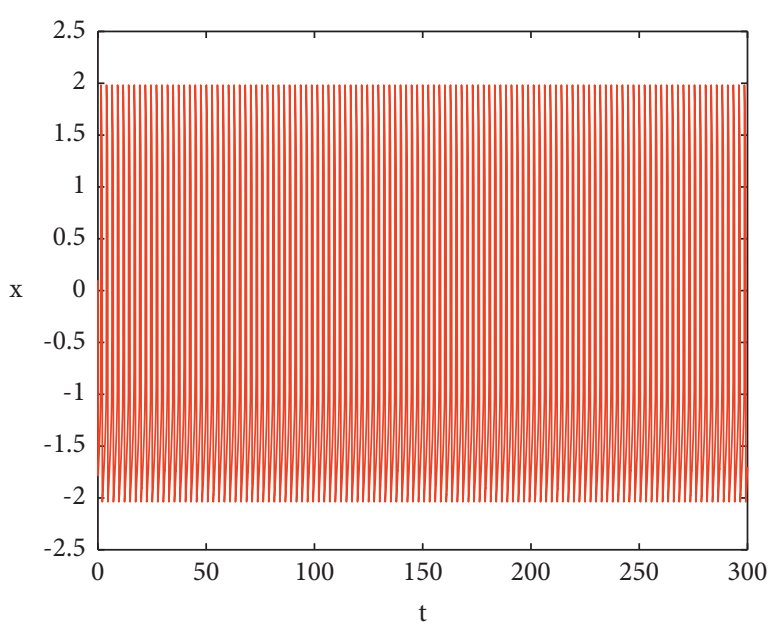

(a)

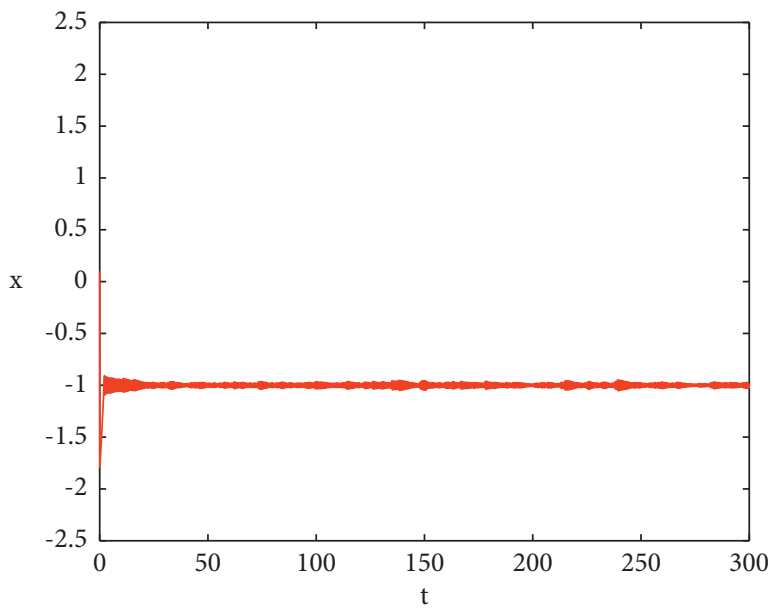

(c)

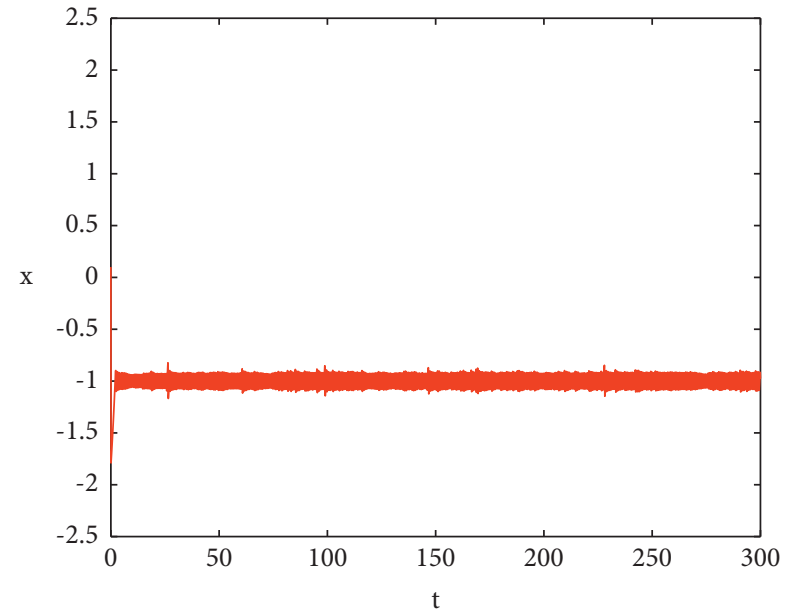

(b)

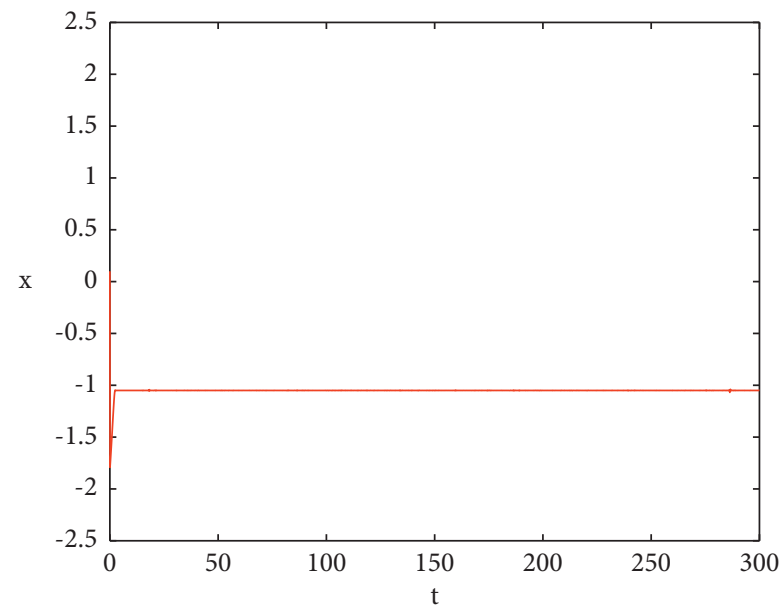

(d)

Figure 2: The temporal evolution of neuronal membrane potential in FHN with different parameters of $a$. (a) $a=0.8$; (b) $a=1.0$; (c) $a=1.0005$; (d) $a=1.05$.

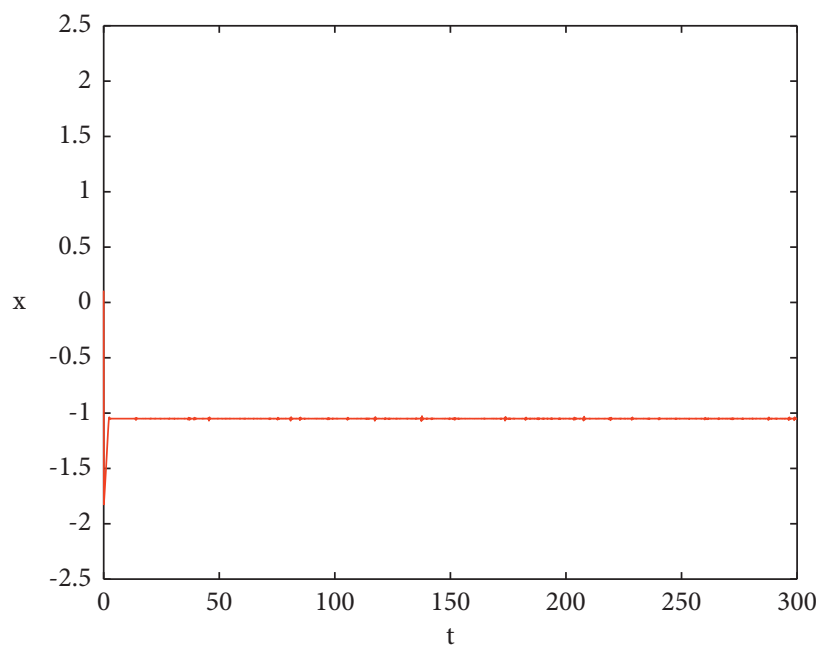

FIgURE 3: Temporal evolution of membrane potential $x$ in FHN neuron system after the addition of flux-controlled memristor. 


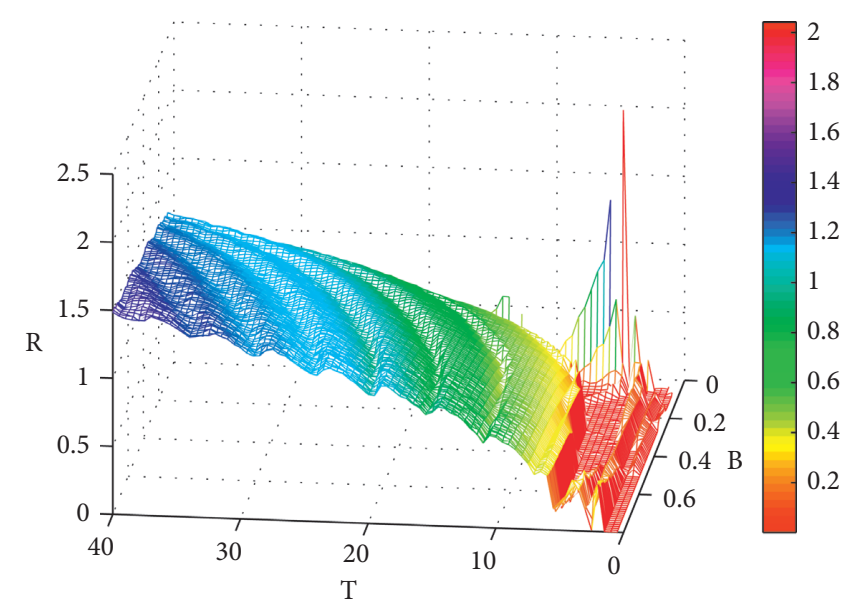

(a)

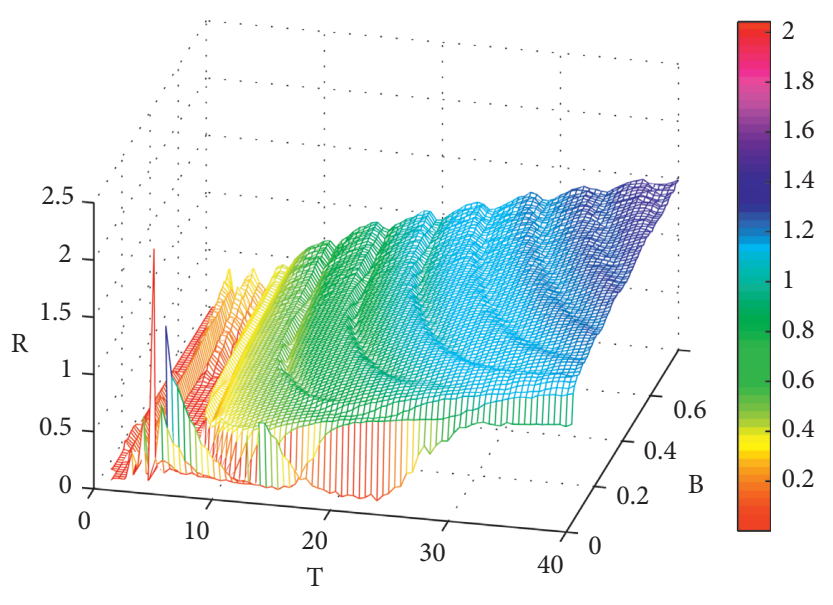

(b)

FIgURE 4: The parameters of the flux-controlled memristor are determined, a three-dimensional surface of FHN neuron system with coherence resonance coefficients $R$ varying with phase noise amplitude $B$ and period $T$. (a) Top view; (b) Back view.

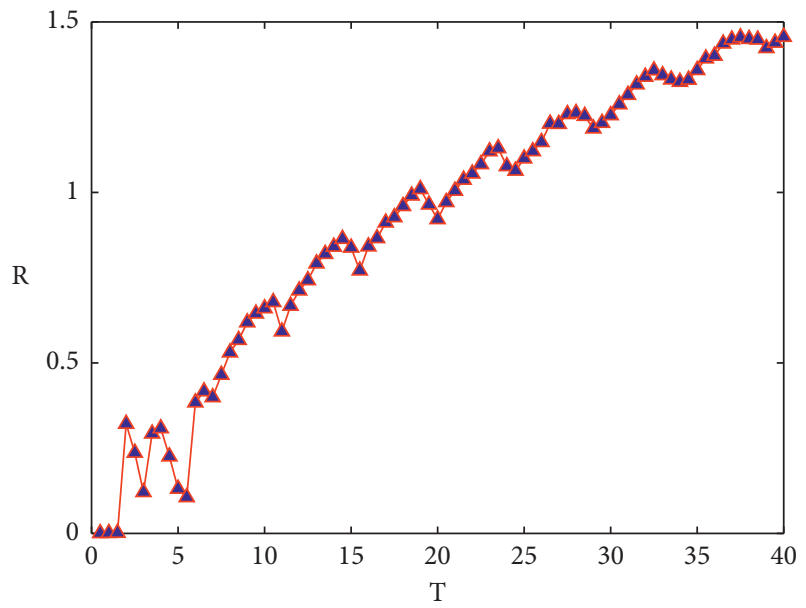

$\neg$ ¿ $\mathrm{B}=0.76$

(a)

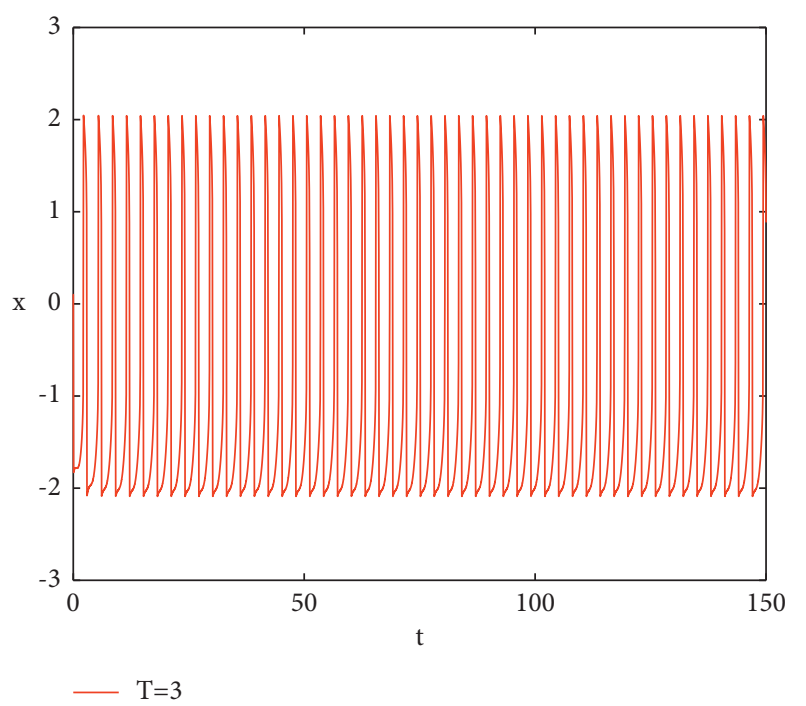

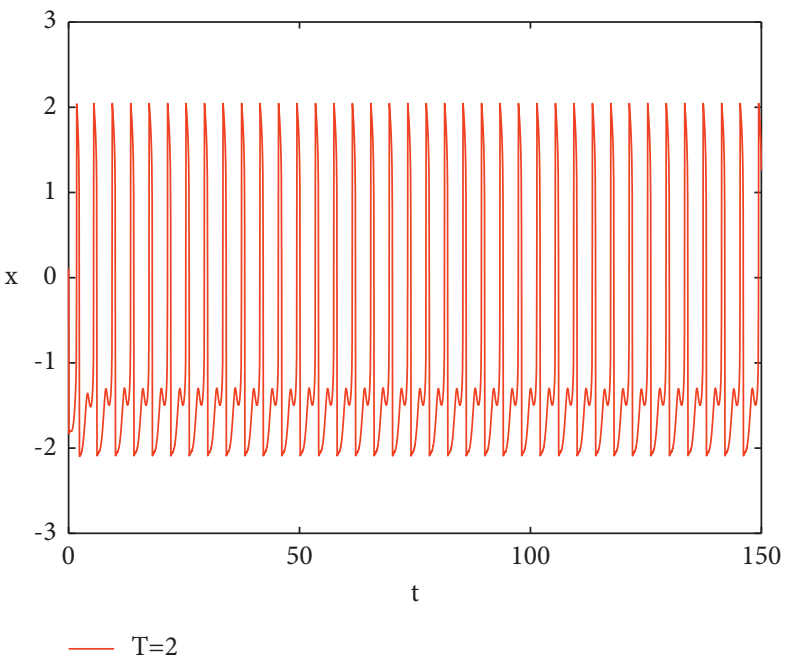

(b)

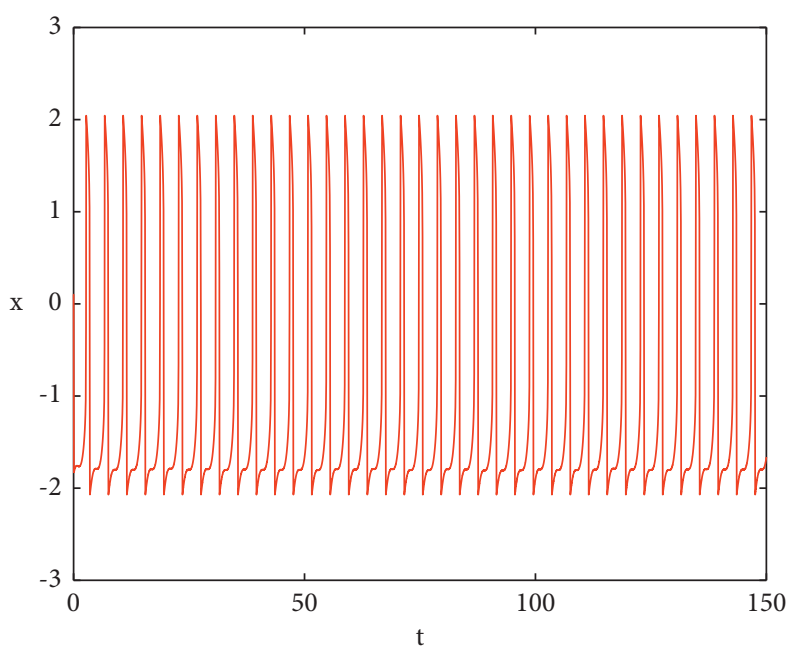

- $\mathrm{T}=4$

(c)

(d)

FIgURE 5: Continued. 


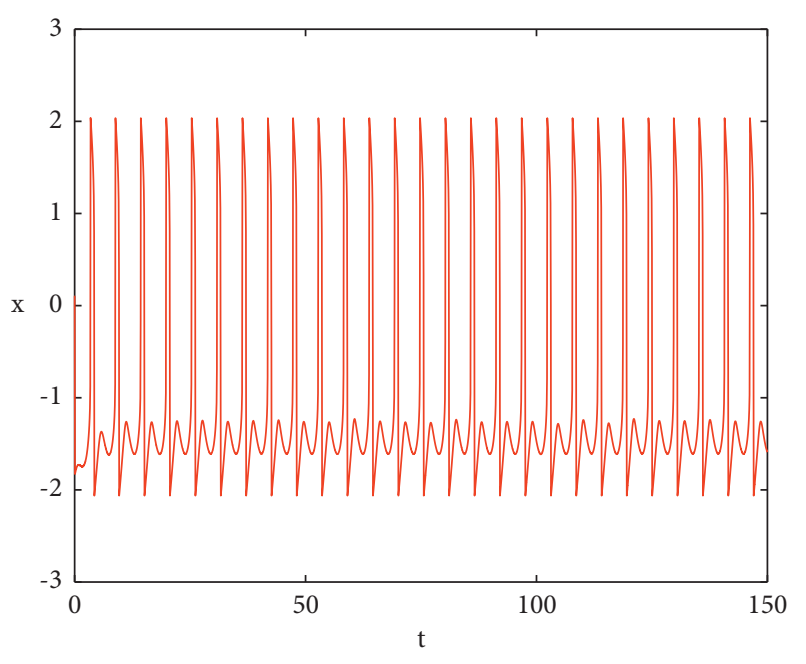

$-\mathrm{T}=5.5$

(e)

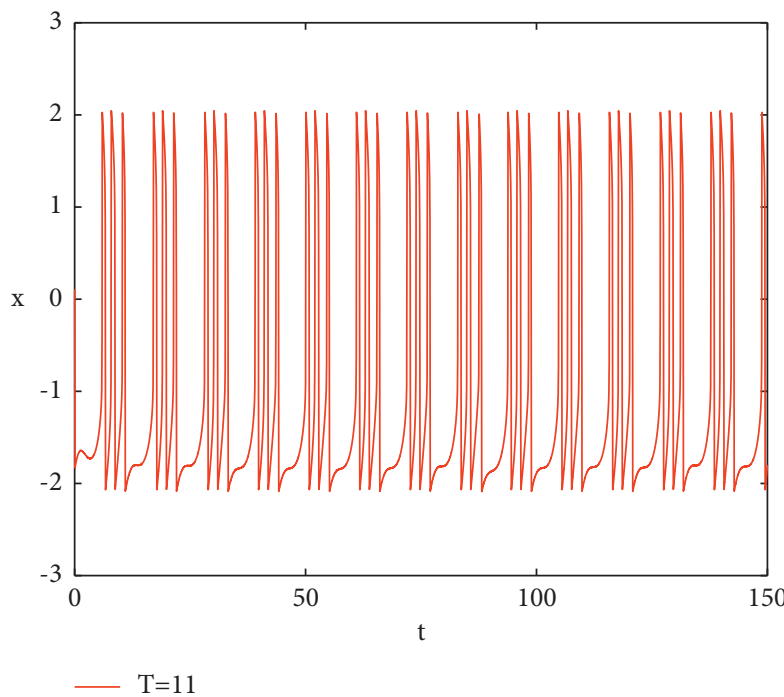

(g)

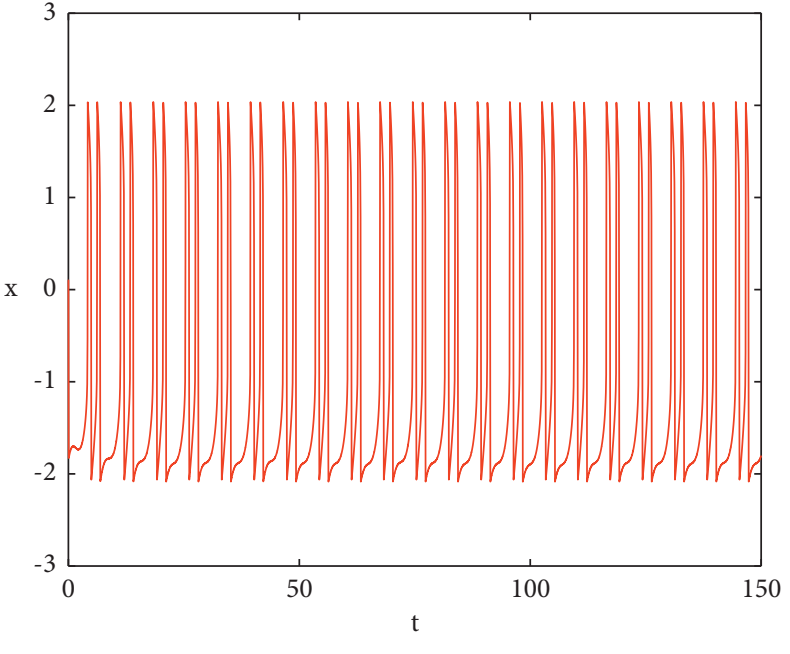

$-\mathrm{T}=7$

(f)

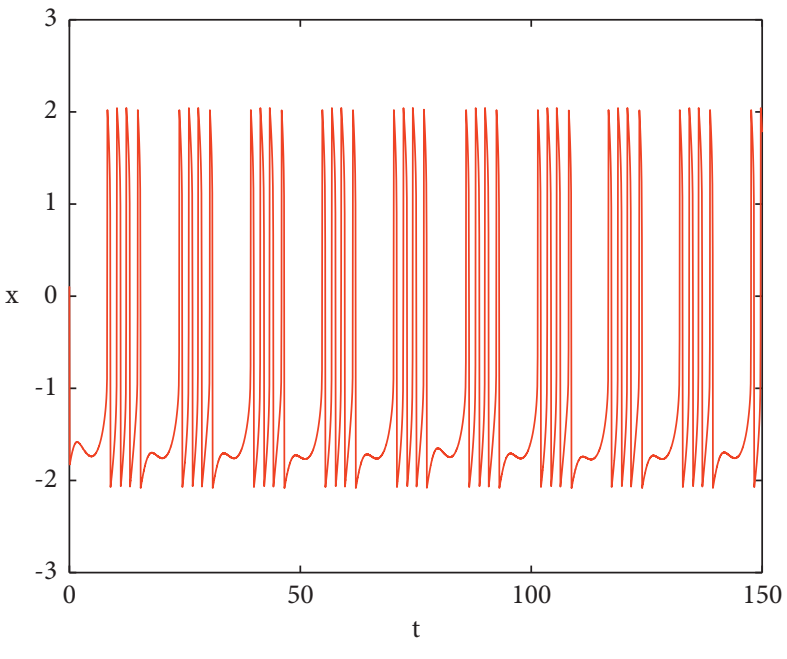

(h)

Figure 5: When phase noise amplitude $B=0.76$, the plot of the coherence resonance coefficient $R$ varying with phase noise period $T$ and the time evolution diagram of the system membrane potential $x$. (a) Variation of $R$ with $T$; (b) $T=2$; (c) $T=3$; (d) $T=4$; (e) $T=5.5$; (f) $T=7$; (g) $T=11$; (h) $T=15.5$.

regular change in FHN neuron membrane potential, similar to the discharging rhythm of period 1 peak discharge. In Figure 5(e), the change of FHN neuron membrane potential was accompanied by the alternating transition between resting state and peak discharge state, that is, the neurons showed cluster discharge behavior similar to period 1 at this time. As shown in Figure 5(b) and (d), when the phase noise period $T=2$ and $T=4$, the degree of discharge regularity shown by the FHN neuron membrane potential is poor compared with that at the two valleys $T=3$ and $T=5.5$. As shown in Figure 5(e) to (h), it is found that as the period number of phase noise increases, the FHN neuron system shows periodic cluster discharge rhythms corresponding to period 1 , period 2 , period 3 and period 4 respectively when the period value $T$ of the relevant resonance coefficient $R$ at the trough is selected as $T=5.5, T=7, T=11$ and $T=15.5$. At the valleys not shown in the figure, the number of periods of the cluster discharge rhythm of the FHN neuron system increases successively by 1 .

Similarly, set the phase noise period $T=8.5$, and select the phase noise amplitude $B$ as the system variation parameter to analyze the coherence bi-resonance behavior and the change of the discharge regularity degree of FHN neuron system. As shown in Figure 6(a), two valleys appear with the increase of phase noise amplitude $B$, and the coherence resonance coefficient value $R$ at the first valley is significantly smaller than that at the second valley. As shown in Figure $6(\mathrm{~b})$, when the phase noise amplitude $B=0.05$, the system changes from the resting state or subthreshold oscillation state to the nerve pulse discharge state. With the 


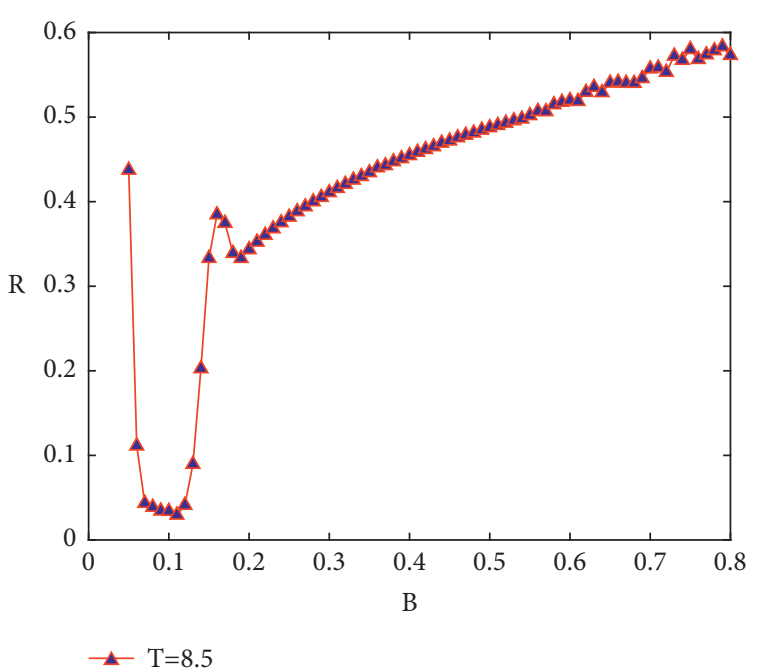

(a)

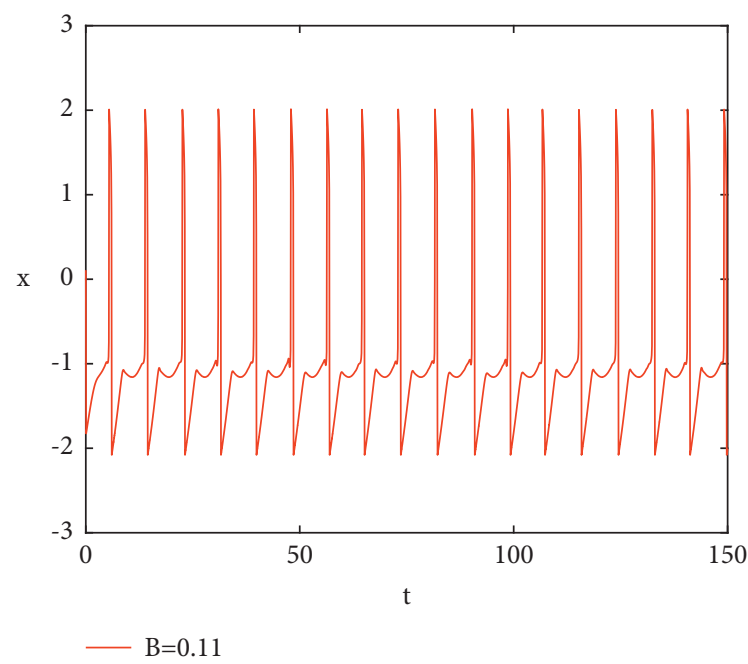

(c)

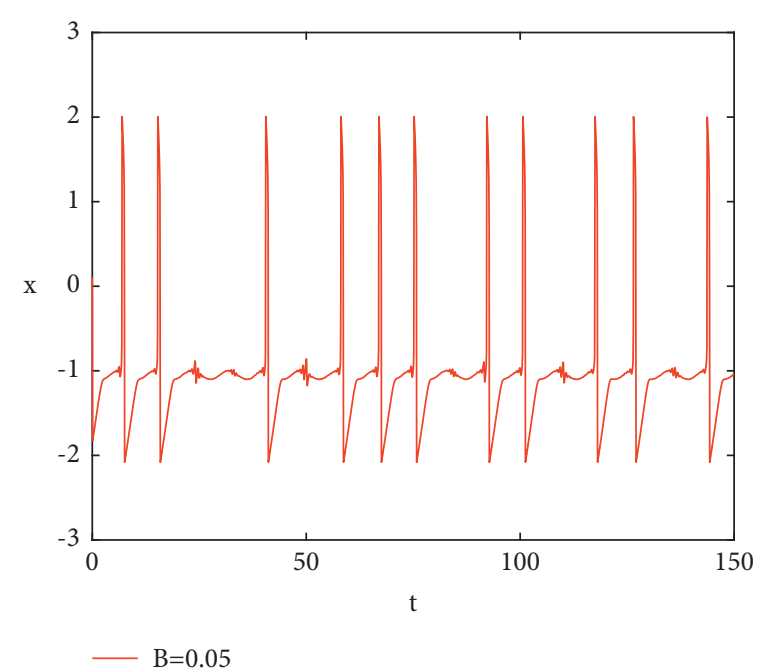

(b)

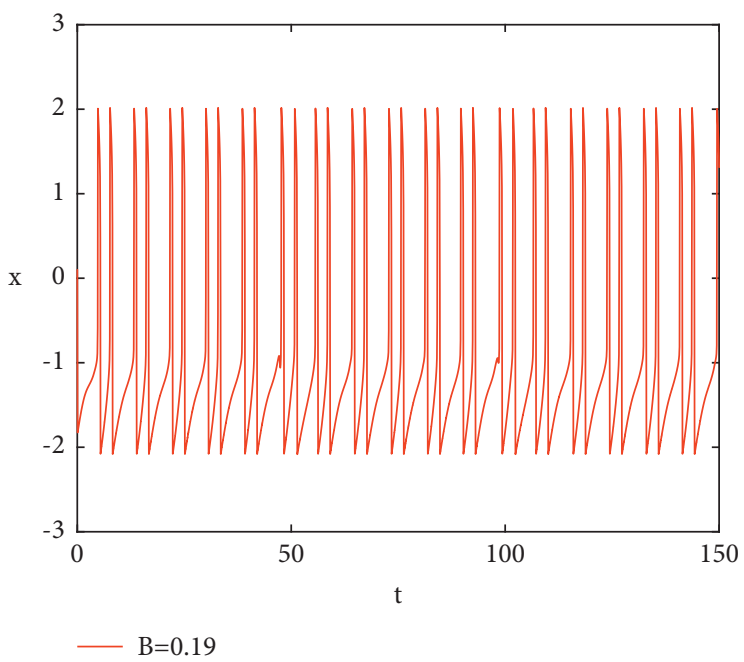

(d)

Figure 6: When phase noise period $T=8.5$, the plot of the coherence resonance coefficient $R$ varying with phase noise amplitude $B$ and the time evolution diagram of the system membrane potential $x$. (a) Variation of $R$ with $B$; (b) $B=0.05$; (c) $B=0.11$; (d) $B=0.19$.

evolution of time, the system appears random and relatively irregular pulse discharge. As shown in Figure 6(c), when the phase noise amplitude $B=0.11$, the system is in the discharge mode of period 1 , the discharge is more regular, and the degree of coherence resonance is the best. As shown in Figure $6(\mathrm{~d})$, when the phase noise amplitude $B=0.19$, the second relatively small valley appears in the curve of coherence resonance coefficient $R$, and coherence resonance behavior occurs again in the neuronal system. Compared with $B=0.11$, the discharge regularity of the system is relatively poor, showing a discharge mode similar to period 2 but slight burr in the discharge process, causing a small difference in the interval of neuronal discharge spikes. Therefore, the value of coherence resonance coefficient $R$ is greater than that at the first valley, and the degree of discharge regularity is relatively poor.
3.2. Coherence resonance behavior of FHN neurons under the variation of phase noise amplitude $B$ and noise intensity $D$. In order to analyze the coherence resonance phenomenon of FHN neurons under the variation of phase noise amplitude $B$ and noise intensity $D$, we still set the flux-controlled memristor parameters $k=0.1, k_{1}=0.2, k_{2}=0.8, \alpha=0.4$ and $\beta=0.02$, set the phase noise period $T=8$, the phase noise amplitude $B$ of the FHN neuron system $B \in[0,0.8]$ and the noise intensity $D \in[0,0.8]$ in this section. It is found that the FHN neuron system shows dynamic behaviors of coherence mono-resonance and coherence bi-resonance. After the addition of the flux-controlled memristor, FHN neurons still remain in a resting state, and the time evolution of their membrane potential $x$ is the same as that shown in Figure 3 .

In Figure 7, when the phase noise amplitude $B \in[0,0.8]$ and the noise intensity $D \in[0,0.8]$, the FHN neuron system 
shows coherence mono-resonance and coherence bi-resonance behaviors. As shown in Figure 7(a), when a certain noise intensity $D$ is selected as the reference point, the value of the coherence resonance coefficient $R$ decreases sharply at first and then reaches the valley as the the phase noise amplitude $B$ gradually increases within the value range. After passing through the valley in a small area, the value of $R$ increases sharply and finally shows a slow upward trend. Similarly, when a certain phase noise amplitude $B$ is selected as the reference point, as the phase noise intensity $D$ gradually increases in the value range, calculate the coherence resonance coefficient $R$ of FHN neuron system. It can be seen that when the phase noise amplitude $B$ is small, FHN neuron system has obvious coherence resonance behavior; when the phase noise amplitude $B$ is large, the coherence resonance coefficient tends to increase smoothly, that is, there is no coherence resonance behavior in FHN neuron system. As shown in Figure. 7(b), when the phase noise intensity $D \in[0,0.03)$ and the phase noise amplitude $B$ increases successively, only one valley appears in the coherence resonance coefficient $R$, that is, the curve of coherence resonance coefficient $R$ firstly decreases to the valley and then increases. Therefore, when the noise intensity $D \in[0,0.03), F H N$ neuron system only shows coherence mono-resonance behavior. When the phase noise intensity $D \in[0.03,0.8]$, the coherence resonance coefficient $R$ drops sharply to the first valley, then climbs rapidly from the valley to the first peak, then drops slowly to the second valley, and then rises slowly from the valley. From an overall perspective, in the region of the first valley, with the gradual increase of the phase noise intensity $D$, the coherence resonance coefficient $R$ at the valley also shows a gradual increase trend. Similarly, the second valley also shows a slow increase trend. When the phase noise intensity $D \in[0.03,0.8]$, the FHN neuron system shows coherence biresonance. Take the phase noise amplitude $B$ as the independent variable, with the increase of the noise intensity $D$, the distance between the two valley of coherence bi-resonance increases gradually, and the discharge regularity degree of the system is lower than that of small noise intensity. When the phase noise amplitude $B \in[0.5,0.7)$, the system also shows coherence resonance behavior. When the amplitude $B$ is in other interval, no obvious coherence resonance phenomenon appears in the FHN neuron system. In conclusion, with the phase noise intensity $D$ as the reference, the coherence resonance or coherence bi-resonance phenomenon of FHN neuron system becomes more obvious with the gradual increase of phase noise amplitude $B$.

In order to verify the correctness of the above conclusions, as shown in Figure 8, the phase noise intensity was selected as $D=0.02$ and $D=0.6$. With the change of phase noise amplitude $B$, FHN neuron system shows coherence mono-resonance and coherence bi-resonance. As shown in Figure $8(\mathrm{a})$, when the phase noise intensity $D=0.02$, the coherence resonance coefficient $R$ increases with the phase noise amplitude $B$, and a valley appears, that is, the coherence mono-resonance phenomenon appears in the system. As shown in Figure 8(b), when the phase noise amplitude $B=0.05$, the membrane potential of FHN neuron system shows irregular random discharge rhythm with the evolution of time, and the regularity of the system is the worst at this time. As shown in Figure 8(c), when the phase noise amplitude $B=0.14$, the value of amplitude $B$ is just at the valley, that is, the value of coherence resonance coefficient $R$ is the smallest. At this time, the system discharge pattern is the most regular, and the neuronal system shows a cluster discharge rhythm similar to period 1 . As shown in Figure $8(\mathrm{~d})$, when the phase noise amplitude $B=0.7$, FHN neurons show a cluster discharge rhythm similar to period 2 . Because the calculation of coherence resonance coefficient $R$ is closely related to the interval of discharge spikes, that is, the cluster discharge regularity of period 2 is weaker than that of period 1. As shown in Figure 8(e), when the phase noise intensity $D=0.6$, the coherence resonance coefficient $R$ increases with the phase noise amplitude $B$, and two valleys appear, that is, the coherence bi-resonance phenomenon occurs in the system. The discharge state of the system at the beginning of pulse generation, the discharge state at the first valley and the discharge state at the second valley are analyzed respectively. As shown in Figure 8(f), when the phase noise amplitude $B=0.04$, the system starts to generate discharge pulses due to the action of noise. At this time, the discharge state of the system is extremely unstable with strong randomness. As the phase noise amplitude increases, as shown in Figure $8(\mathrm{~g})$, and when the phase noise amplitude $B=0.08$, the coherence resonance coefficient $R$ is in the first valley, that is, the minimum value of $R$ is obtained. At this time, the neuron system shows a cluster discharge rhythm similar to period 1 , so the discharge pattern is the most regular. As shown in Figure $8(\mathrm{~h})$, when the phase noise amplitude $B=0.21$, it was found that although the discharge rhythm of the neuron system was similar to that of period 2, it includes some irregular discharge spikes. Therefore, the discharge regularity of the neuron system was inferior to that when the amplitude $B=0.08$.

\subsection{Coherence resonance behavior of FHN neurons under the} variation of noise intensity $D$ and period $T$ of phase noise. In order to analyze the coherence resonance phenomenon of FHN neurons under the variation of noise intensity $D$ and period $T$ of phase noise. This section readjusts the parameter values of flux-controlled memristor to $k=0.08, k_{1}=0.2$, $k_{2}=0.8, \alpha=0.4$ and $\beta=0.02$. In FHN neuron system, set the phase noise amplitude $B=0.05$, the phase noise intensity $D \in[0,4]$ and the period $T \in[0,40]$. It is found that FHN neural system exhibits dynamic behaviors of coherent mono-resonance, coherent bi-resonance and coherent multi-resonance. As shown in Figure 9, after setting $D=$ 0.001 and $T=0.1$ on the basis of the above parameter values and adding the influence of the flux-controlled memristor, it can be found from the time evolution diagram of membrane potential $x$ that FHN neurons still maintain the state of resting discharge.

As shown in Figure 10, the FHN neuron system exhibits coherence bi-resonance and coherence multi-resonance behaviors when the phase noise intensity $D \in[0,4]$ and the 


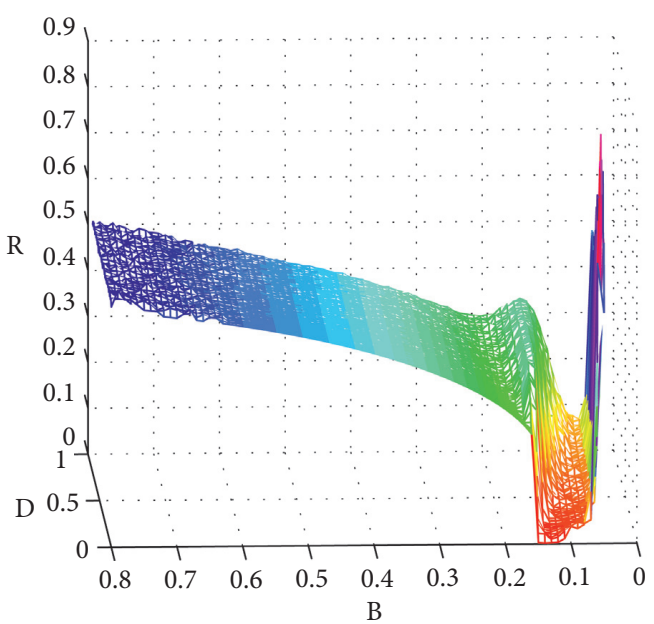

(a)
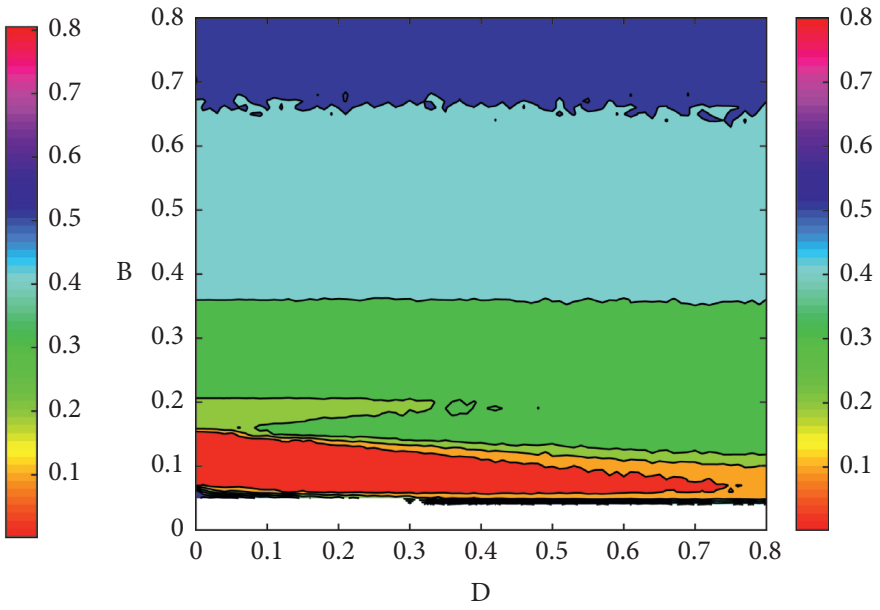

(b)

FiguRE 7: When flux-controlled memristor parameters are determined, the plot of coherent resonance coefficient $R$ of FHN neuron system varying with phase noise amplitude $B$ and noise intensity $D$. (a) 3D view; (b) The floor plan.

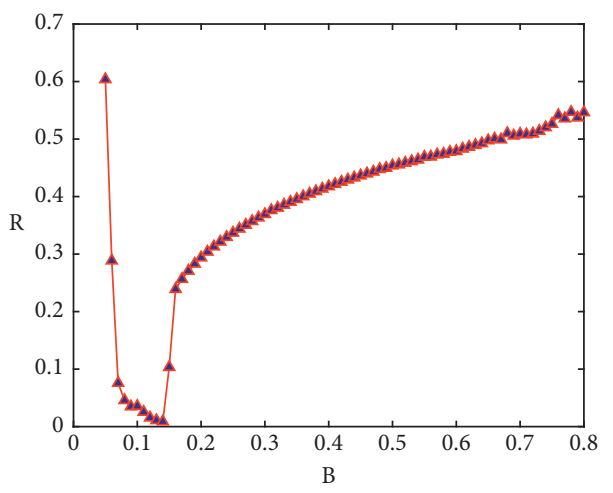

$\neg \mathrm{D}=0.02$

(a)

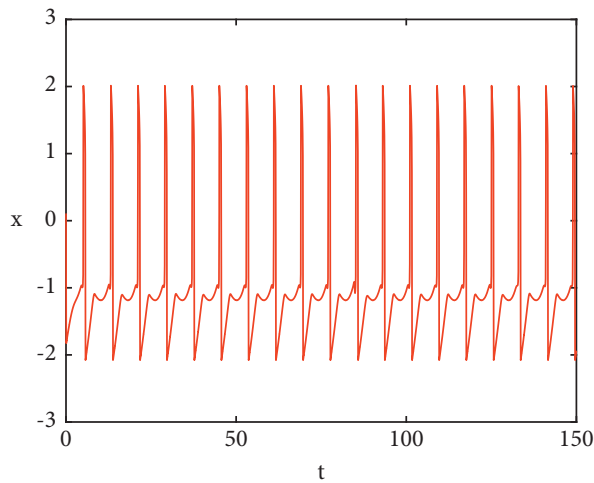

$-\mathrm{B}=0.14$

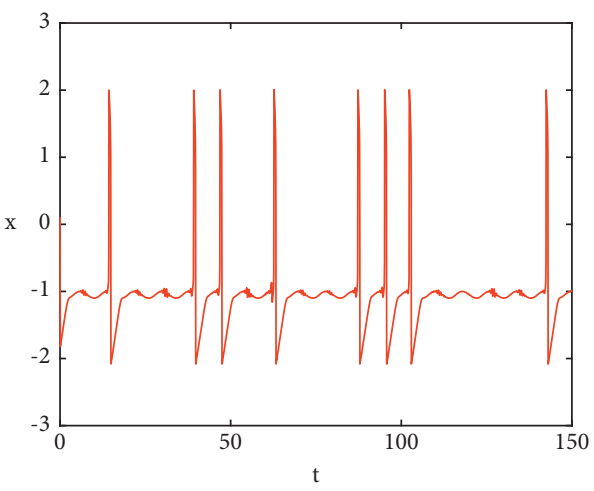

— $\mathrm{B}=0.05$

(b)

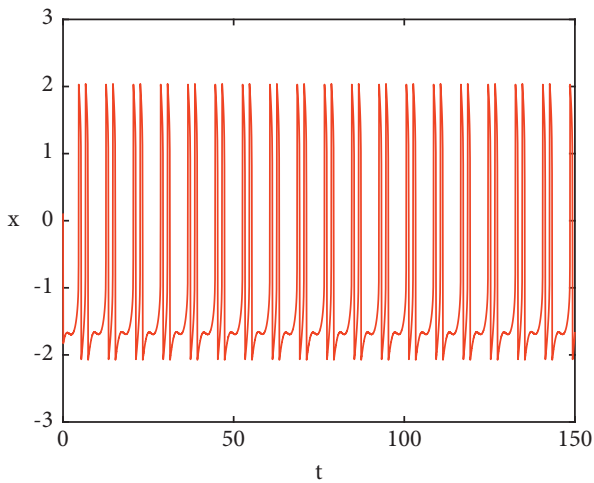

- $\mathrm{B}=0.7$

(c)

(d)

Figure 8: Continued. 


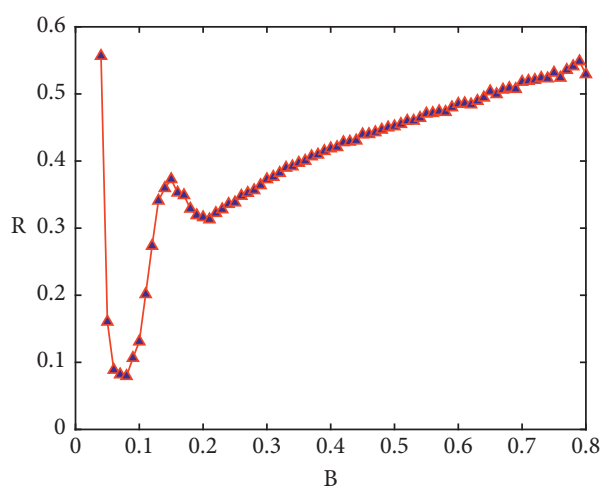

$\star \mathrm{D}=0.6$

(e)

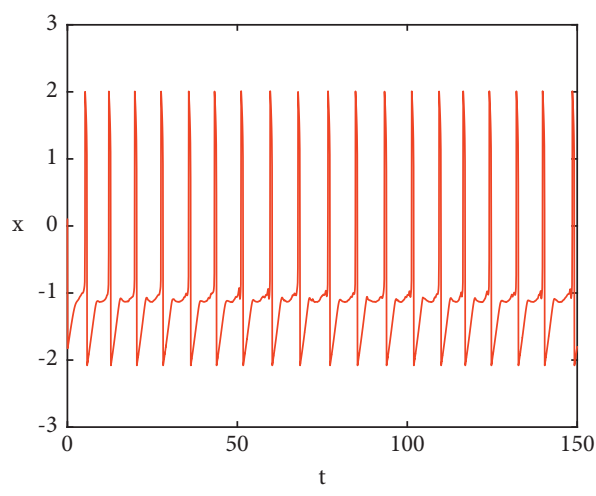

- $\mathrm{B}=0.08$

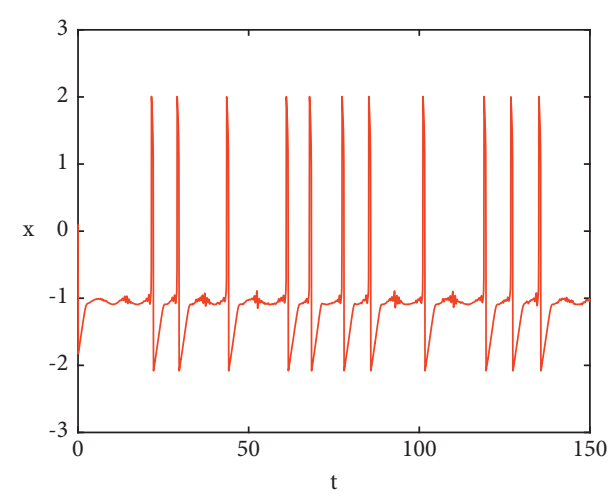

- $\mathrm{B}=0.04$

(f)

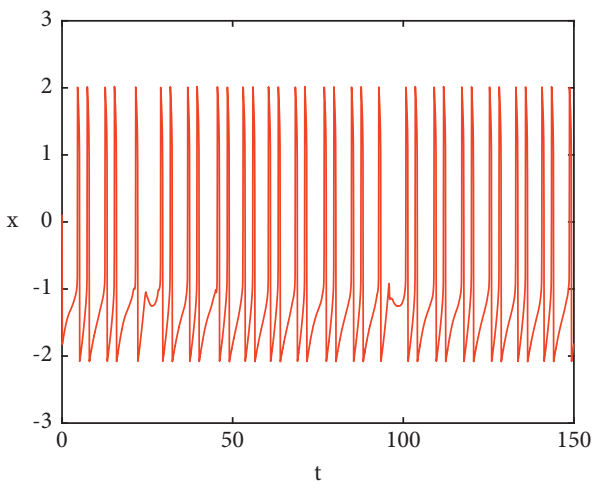

- $\mathrm{B}=0.21$

(g)

(h)

FIGURE 8: When the phase noise intensity changes, the plot of the coherence resonance coefficient $R$ varying with phase noise amplitude $B$ and the time evolution diagram of the system membrane potential $x$. (a)When $D=0.02$, variation of $R$ with $B$; (b) $D=0.02, B=0.05$; (c) $D=0.02, B=0.14$; (d) $D=0.02, B=0.7$; (e)When $D=0.6$, variation of $R$ with $B$; (f) $D=0.6, B=0.04$; (g) $D=0.6, B=0.08$; (h) $D=0.6, B=0.21$.

period $T \in[0,40]$. As shown in Figure 10(a), in the twoparameter variation process of noise intensity $D$ and period $T$ of phase noise, FHN neuron system exhibits different coherence resonance behaviors in different parameter regions. Firstly, with the phase noise intensity $D$ as the reference analysis, FHN neurons show rich dynamic behaviors such as coherence mono-resonance, bi-resonance and multiresonance with the gradual increase of phase noise period $T$. When noise intensity $D \in[0,0.3)$, FHN neurons show coherence mono-resonance behavior in the change process of phase noise period $T$, and the value of coherence resonance coefficient $R$ at the valley of the system is relatively small in the whole measurement range of noise intensity $D$, which shows that the coherence resonance behavior of FHN neuron system is more obvious and the regularity of the system discharge is higher. When the phase noise intensity $D \in[0,0.3)$, with the change of period $T$, the coherence resonance coefficient $R$ of FHN neuron system shows a smaller value in the larger range of period $T$, and the range of wave valley is the largest. When the noise intensity $D \in[0.3,1.9)$, FHN neuron system shows coherence biresonance and coherence multi resonance behavior with the gradual increase of phase noise period $T$. Most of the value ranges of noise intensity show coherence bi-resonance behavior, that is, the curve of coherence resonance coefficient $R$ has two valleys. The coherence multi resonance behavior occurs intermittently when the noise intensity $D \in[0.3,1.9)$. For example, when the phase noise intensity $D=0.5$ and $D=0.7$, with the gradual change of the phase noise period $T$, the system has three valleys. The value of the coherence resonance coefficient $R$ at the first valley is greater than that at the second and third valleys, indicating that the order of the system is poor at this time, the values of the coherence resonance coefficients $R$ at the second and third wave valleys are basically the same and relatively small. The smaller the value of $R$, the higher the regularity and order of the neuron system. That is, when the coherence resonance coefficient $R$ of the system is at the wave valley, the value of the phase noise period $T$ is larger, and the coherence resonance behavior of the system is more obvious. When the noise intensity $D \in[1.9,4]$, the FHN neuron system shows coherence bi-resonance and coherence multi resonance behavior. Specifically, when the noise intensity $D \in[1.9,3.3)$, and is set different values, the system 


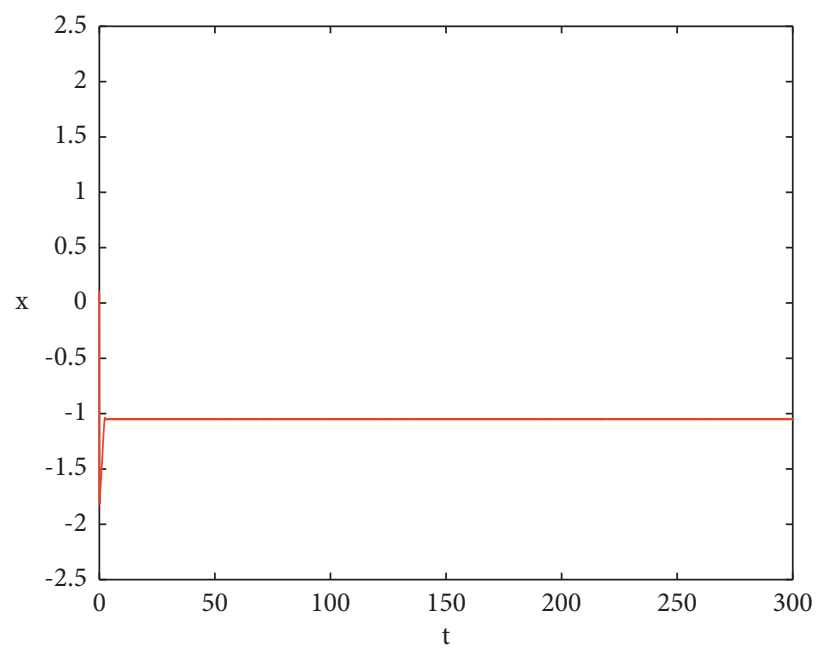

Figure 9: Temporal evolution of membrane potential $x$ in FHN neuron system after the addition of flux-controlled memristor.

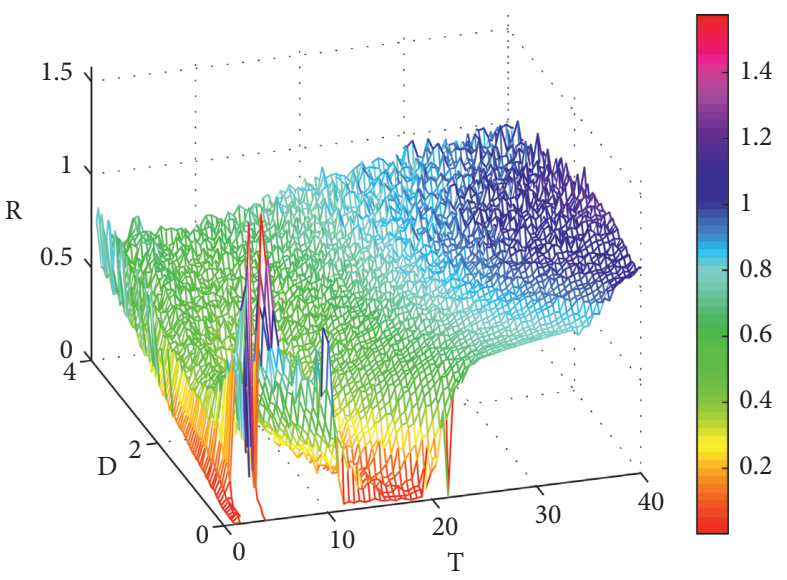

(a)

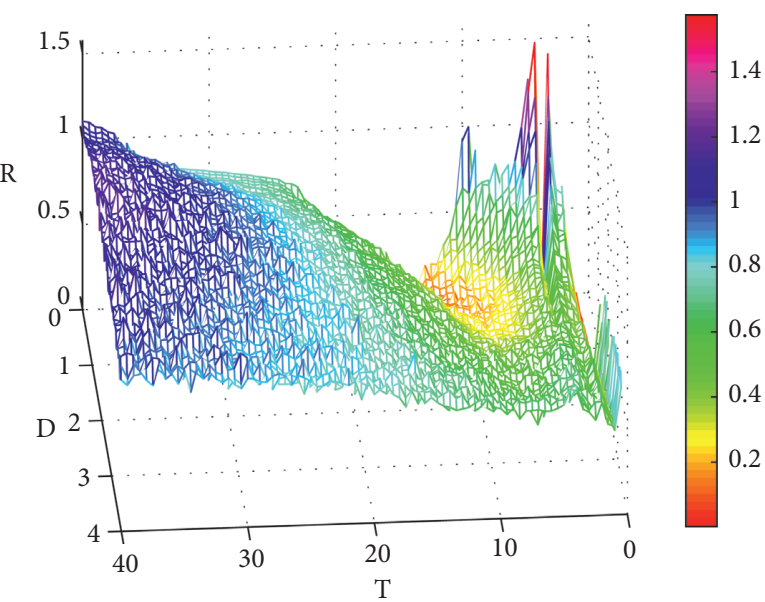

(b)

FiguRE 10: When the parameters of flux-controlled memristor are determined, the periodic variation diagram of coherence resonance coefficient $R$ of FHN neuron system with noise intensity $D$ and period $T$ of phase noise. (a) Top view; (b) Back view.

increases a discontinuous valley when the phase noise period $T$ is small. That is, the system has two or three obvious valleys, which appear alternately and discontinuous. When the noise intensity $D \in[1.9,3.3)$, with the change of the phase noise period of the independent variable $T$, the system shows complex dynamic behavior. When the noise intensity $D \in[3.3,4]$, the system has three obvious valleys, at this time, the system shows coherence multi resonance behavior, in which the value of coherence resonance coefficient $R$ at the first valley is relatively smaller than those at the second and third troughs; then, take the phase noise period $T$ as the benchmark for analysis, it is found that with the gradual increase of the phase noise intensity $D$, FHN neurons show the dynamic behavior of coherence mono-resonance and no obvious resonance. When the phase noise period $T \in[0,3)$ and $T \in[15,40]$, the curve of coherence resonance coefficient $R$ basically shows an upward trend, and there is no obvious valley, that is, within the value range of the period, there is no obvious coherence resonance phenomenon with the change of phase noise intensity $D$. When the phase noise period $T \in[3,15)$, with the gradual increase of the phase noise intensity $D$, the coherence resonance coefficient $R$ has mass valley, and there is only one valley, that is, the system has only coherence mono-resonance behavior in this period. From the overall point of view, when the phase noise intensity is used as the benchmark to analyse, with the gradual increase of the phase noise intensity, the first valley basically does not appear in the first half of the region. Similarly, the second valley also shows the same behavior, while the third valley shows special phenomena. Firstly, when the noise intensity $D$ is small and the curve of coherence resonance coefficient $R$ appears a wide range of valley with the increase of phase noise period $T$, the corresponding period $T$ of phase noise is the largest and the coherence resonance behavior is more abundant; Secondly, when the noise intensity $D$ increases gradually, the curve of coherence resonance 


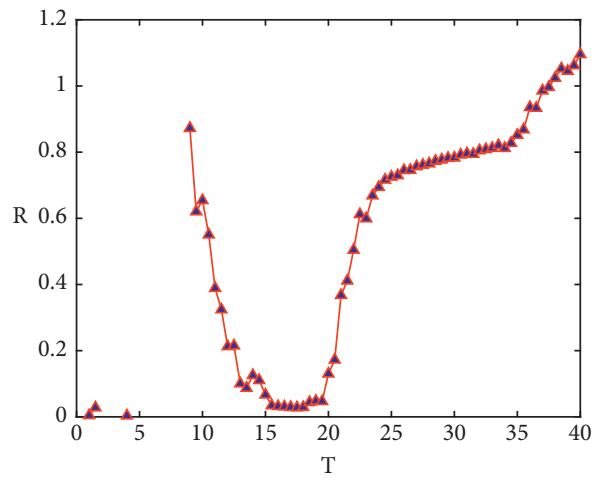

$₫ \mathrm{D}=0.05$

(a)

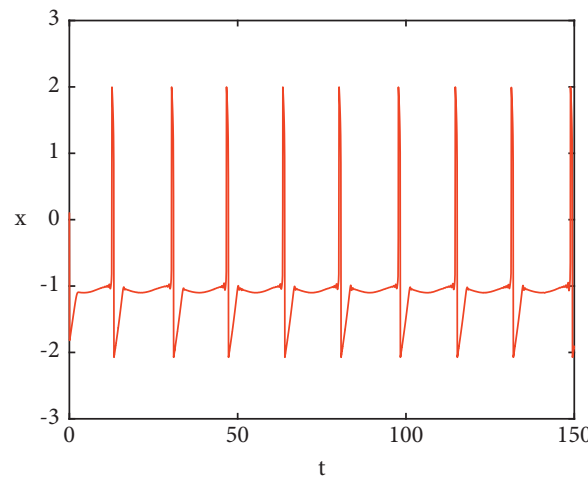

- $\mathrm{T}=17$

(c)

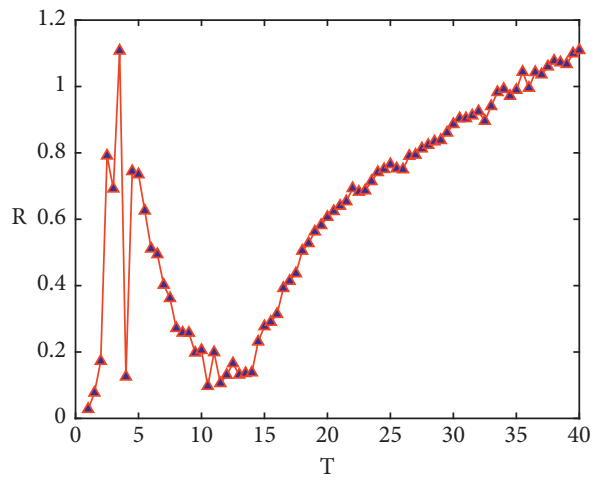

^ $\mathrm{D}=0.5$

(e)

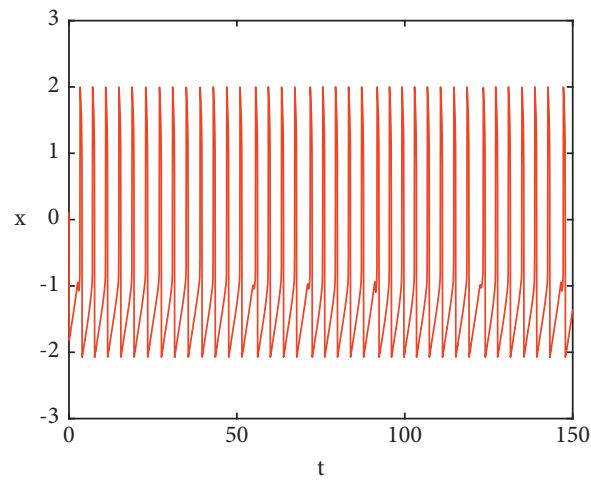

- $\mathrm{T}=4$

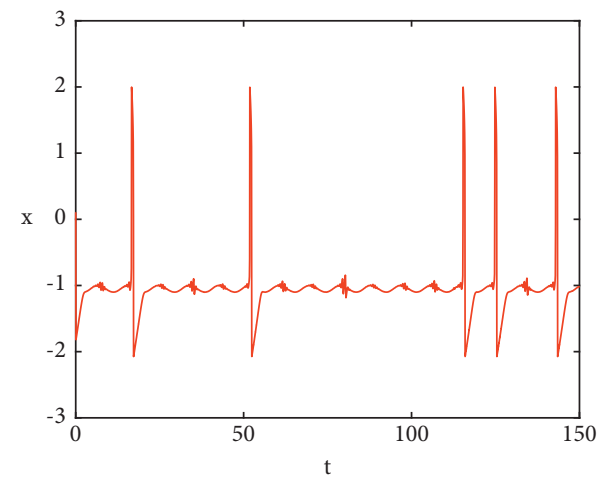

- $\mathrm{T}=9$

(b)

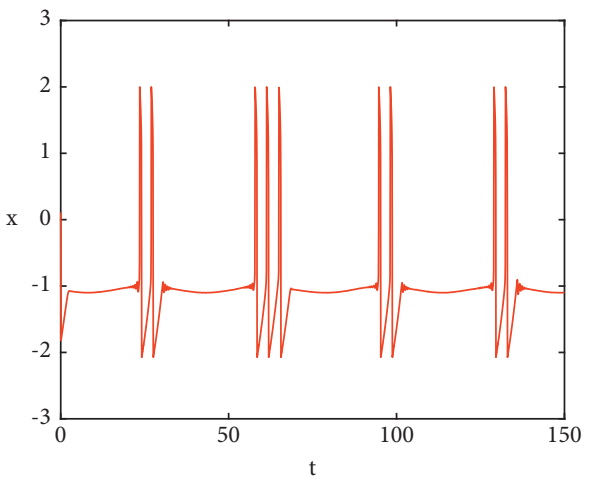

— $\mathrm{T}=35$

(d)

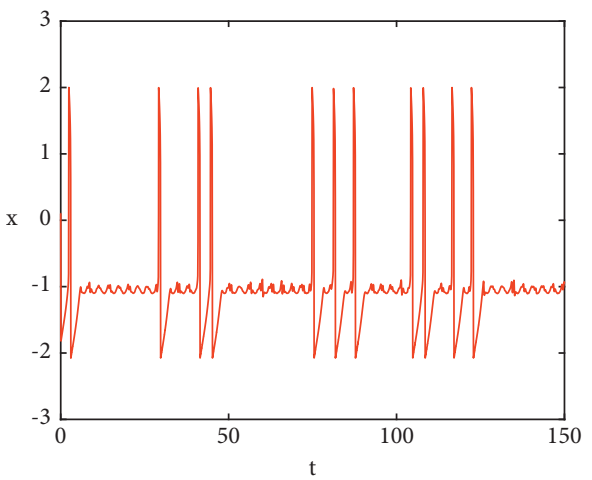

- $\mathrm{T}=3$

(f)

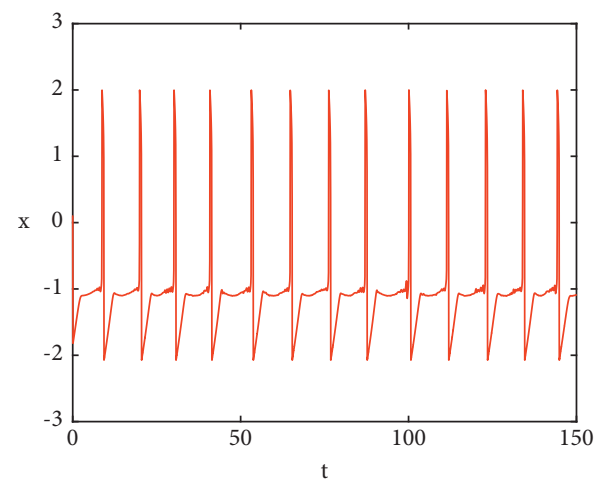

— $\mathrm{T}=11.5$

(h)

(g)

Figure 11: Continued. 


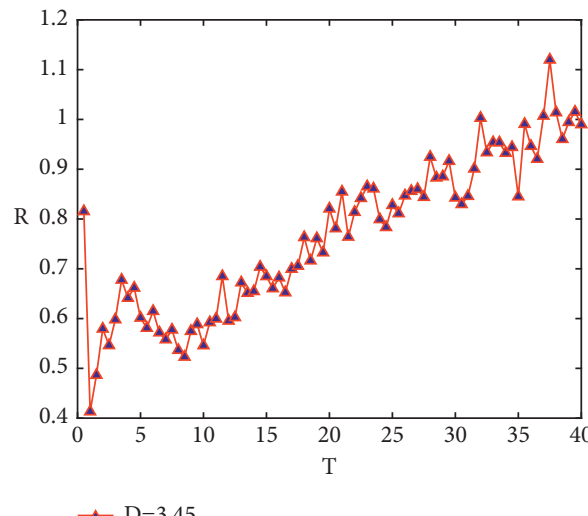

(i)

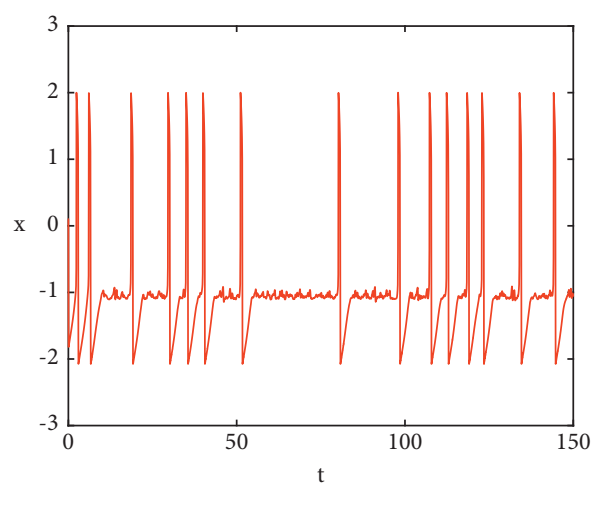

- $\mathrm{T}=2.5$

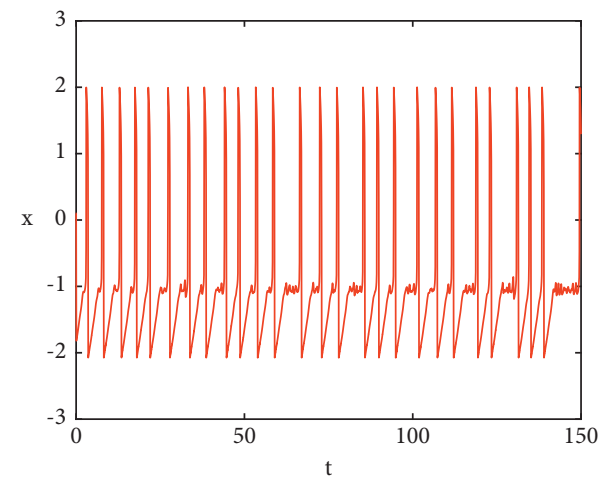

— $\mathrm{T}=1$

(j)

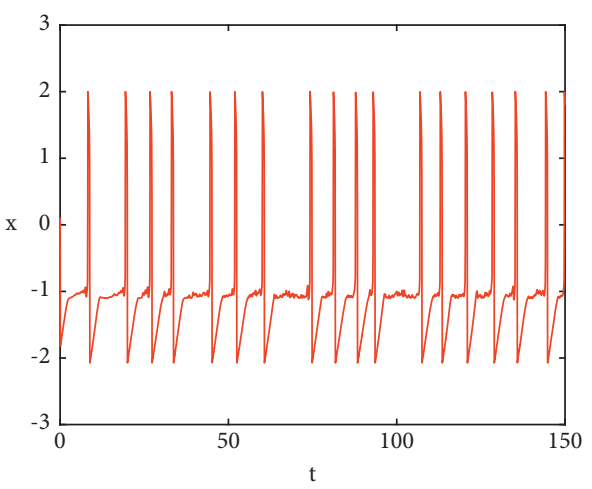

— $\mathrm{T}=8.5$

(k)

(l)

FIGURE 11: When the phase noise intensity varies, the variation of coherence resonance coefficient $R$ with period $T$ of phase noise and the time evolution diagram of the system membrane potential $x$. (a)When $D=0.05$, variation of $R$ with $T$; (b) $D=0.05, T=9$; (c) $D=0.05$, $T=17$; (d) $D=0.05, T=35$; (e)When $D=0.5$, variation of $R$ with $T$; (f) $D=0.5, T=3$; (g) $D=0.5, T=4$; (h) $D=0.5, T=11.5$; (i)When $D=3.45$, variation of $R$ with $T$; (j) $D=3.45, T=1$; (k) $D=3.45, T=2.5$; (l) $D=3.45, T=8.5$.

coefficient $R$ appears valley with the increase of phase noise period $T$, and the corresponding phase noise period $T$ decreases successively. When the noise intensity $D$ exceeds 2 , the periodic values $T$ of the system entering the trough are basically consistent. When the phase noise period $T$ is used as the benchmark for analysis, the coherence resonance behavior of the system is relatively simple. That is, only in the interval where the period $T$ is small, the coherence resonance behavior of the system appears; when the period $T$ is large, the original order degree of the system is destroyed, then there is no obvious coherence resonance behavior. In conclusion, the FHN neurons exhibit rich coherence resonance behavior during the two-parameter variation of noise intensity $D$ and period $T$ of phase noise.

In order to verify the coherence resonance behavior of FHN neuron system under the variation of noise intensity $D$ and period $T$ mentioned above, Figure 11 takes the value of the discrete phase noise intensity $D$ and gives the curve of the coherence resonance coefficient $R$ with the increase of the period $T$ respectively to verify the coherence monoresonance, bi-resonance and multi-resonance behaviors of the FHN neuron system. Similarly, Figure 12 takes the period value $T$ of discrete phase noise, and shows the curve of coherence resonance coefficient $R$ changing with the increase of noise intensity $D$ to verify the coherence resonance behavior of the nervous system in some regions of the parameter. In Figure 11, when the phase noise intensity $D=0.05, D=0.5$ or $D=3.45$, the coherence resonance coefficient $R$ of the system changes with the increase of the period $T$, as shown in Figure 11(a), (e) and (i) respectively. By comparing the three figures, it can be seen that the corresponding period value of the mass coherence resonance phenomenon in the neuron system decreases with the increase of the noise intensity $D$. However, the corresponding coherence resonance coefficient $R$ increases with the increase of noise intensity $D$. And with the increase of noise intensity $D$, the coherence resonance behavior of the FHN neuron system becomes more complex, and the regularity of the discharge decreases when the coherence resonance phenomenon occurs. Figure11(a) shows that the FHN neuron system exhibits obvious coherence resonance behavior from the variation curve of coherence resonance coefficient $R$. Figure 11(b), (c) and (d) show the time evolution sequence of membrane potential $x$ of FHN neuron system when coherence resonance coefficient $R$ is valued before the valley $T=9$, at the valley $T=17$ and after the 


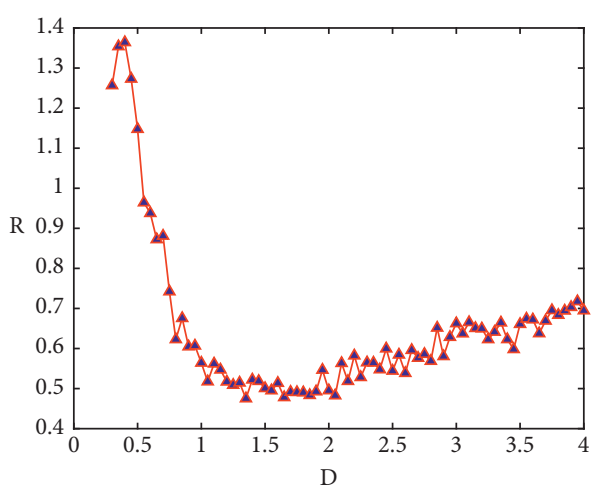

$\leftarrow \mathrm{T}=3.5$

(a)

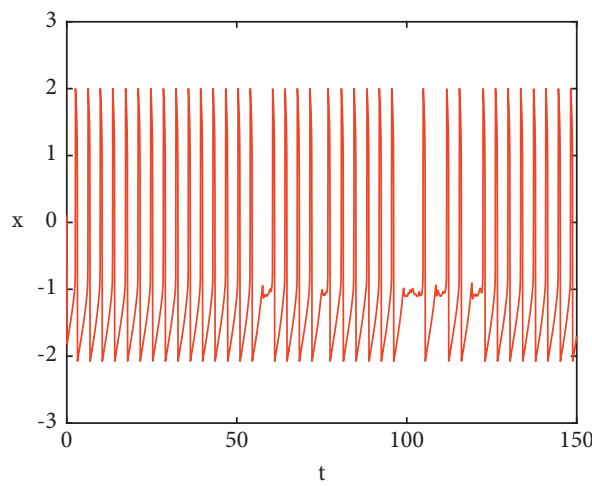

- $\mathrm{D}=1.7$

(c)

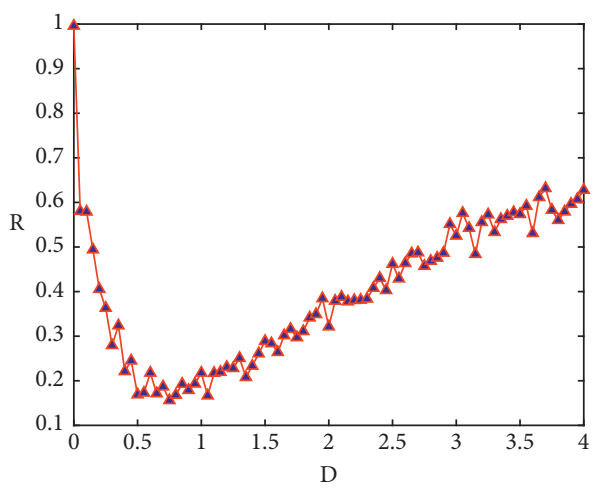

$\star \mathrm{T}=9.5$

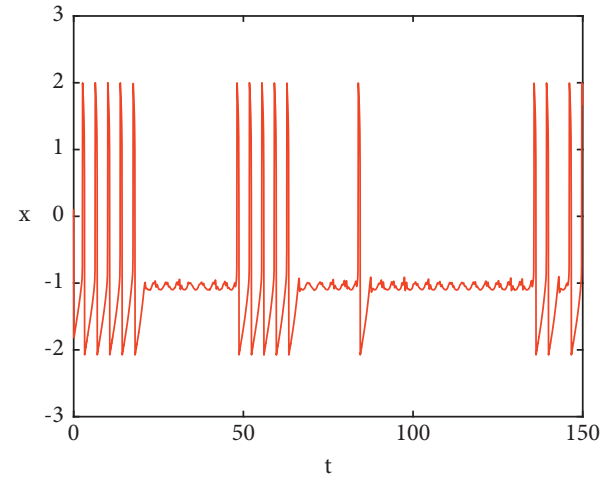

- $\mathrm{D}=0.4$

(b)

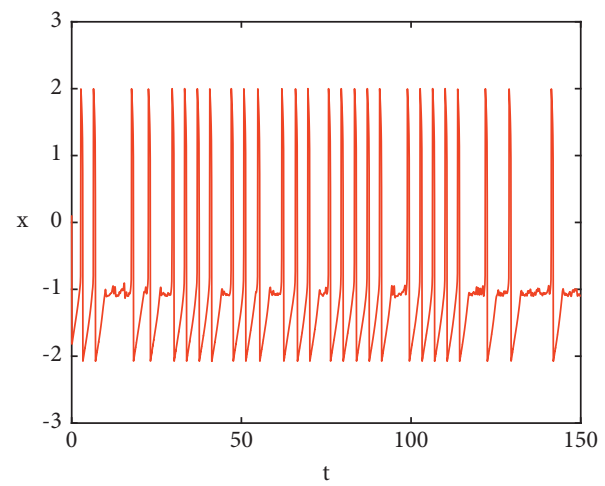

$-\mathrm{D}=3$

(d)

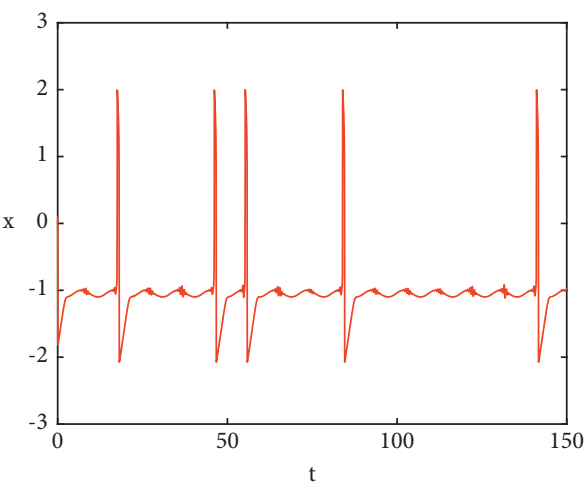

- $\mathrm{D}=0$

(f)

Figure 12: Continued. 


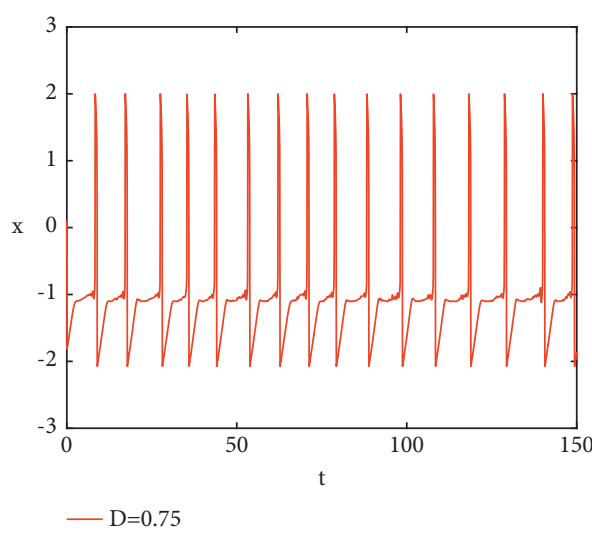

(g)

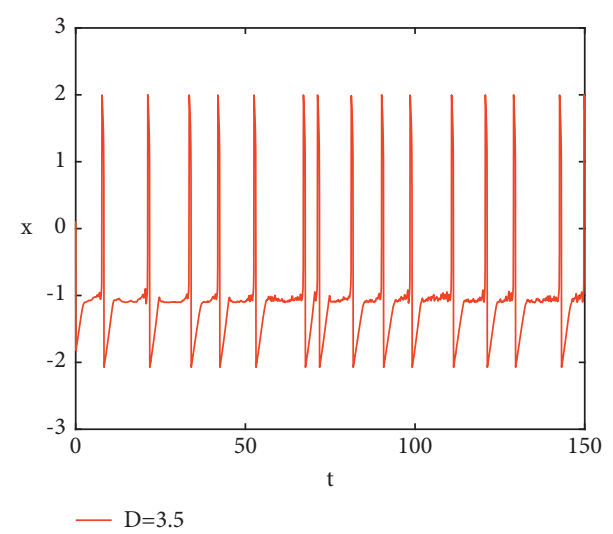

(h)

Figure 12: When the period of phase noise varies, the variation of coherence resonance coefficient $R$ with the phase noise intensity $D$ and the time evolution diagram of the system membrane potential $x$. (a)When $T=3.5$, variation of $R$ with; (b) $T=3.5, D=0.4$; (c) $T=3.5$, $D=1.7$; (d) $T=3.5, D=3$; (e) When $T=9.5$,variation of $R$ with $D$; (f) $T=9.5, D=0$; (g) $T=9.5, D=0.75$; (h) $T=9.5, D=3.5$.

valley $T=35$. Before the valley, $T=9$, the system discharge is sparse and irregular; At the lowest valley, $T=17$, the system discharges uniformly and regularly, and the neuronal system shows a cluster discharge rhythm similar to period 1; After the valley, $T=35$, the system shows the chaotic discharge rhythm where cluster discharges occur crosswise in period 2 and period 3 . And at this time, the regularity and stability of the system discharge are lower than that of the neurons at the valley. As shown in Figure 11(e), it is found from the variation curve of the system coherence resonance coefficient $R$ that the FHN neuron system has coherence multi-resonance. Figure 11(f) (g) and (h) respectively represent the time process diagram of the membrane potential of the FHN neuron system when the periodic value $T$ of the phase noise at the three valleys of the coherence resonance coefficient curve are determined. The period values at the three valleys are $T=3, T=4$ and $T=11.5$, respectively. And the coherence resonance coefficient $R$ at the first valley is significantly greater than those at the second and third valleys, indicating that the discharge regularity degree of the neuronal system at this time is worse than that at the other two valleys. As shown in Figure 11(f), the system discharge is sparse and irregular, with a wide range of alternate resting state and pulse discharge state. In the second and third wave valleys, the coherence resonance coefficients $R$ are basically consistent, indicating that the system is in a relatively regular and orderly discharge state. As shown in Figure 11(g), the system presents the peak discharge rhythm of period 1, but a small part of irregular discharge appears in the discharge sequence. While in Figure 11(h), the system shows a cluster discharge mode similar to period 1 , and the time interval of discharge peaks is more uniform, so the phenomenon of coherence resonance of FHN neuron system is better. As shown in Figure 11(i), it is found from the variation curve of the system coherence resonance coefficient that the FHN neuron system has coherence multi resonance, mainly in the form of two large valleys and multiple small valleys. Figure 11(j) and (l) respectively represent the time evolution process of the system membrane potential at two large valleys. With period value $T=1$ and $T=8.5$ the value of the coherence resonance coefficient $R$ at the valleys is relatively small, and the system discharge is more orderly. Figure $11(\mathrm{k})$ shows the time evolution diagram of the system membrane potential at a small valley. With the period value $T=2.5$, the regularity of the system discharge is significantly worse than those of the former two. In conclusion, by discretely selecting the value of the phase noise intensity $D$, it is verified that the neuron system exhibits rich coherence resonance behavior with the increase of the period $T$.

Similarly, in Figure 12, this section takes the period $T$ of discrete phase noise and gives the curve of coherence resonance coefficient $R$ changing with the increase of noise intensity $D$. FHN neuron system only shows coherence mono-resonance behavior. In this part, when the phase noise period $T=3.5$ and $T=9.5$, the curves of the coherence resonance coefficient $R$ of the system changing with the increase of the noise intensity $D$ are respectively shown. As shown in Figure 12(a) and (e), there is only one large valley in this curve, that is, the system only shows coherence monoresonance behavior. Figure 12(b) (c) and (d) show the noise intensity $D=0.4, D=1.7$ and $D=3$, which are taken respectively from Figure 12(a), the time course of the system's membrane potential $x$ discharge shows different discharge regularity. When the noise intensity $D=0.4$, the coherence resonance coefficient $R$ is at the peak of the curve, and the discharge state of the system is relatively unstable and less regular. When the noise intensity $D=1.7$, the coherence resonance coefficient $R$ is at the valley of the curve, and the system shows orderly discharge state except for small-range irregular discharge. When the noise intensity $D=1.7$, the greater noise intensity value after the valley is selected as a comparison, and the degree of irregularity of the system increases, indicating that the system has the optimal discharge state when the coherence resonance coefficient reaches the minimum value. In Figure 12(f) (g) and (h), when the noise intensity $D=0, D=0.75$ and $D=3.5$, which are taken from Figure 12(e) respectively, the system membrane potential $x$ discharge sequence changes from 
irregular to regular, and then from regular to irregular. When the noise intensity $D=0$, the coherence resonance coefficient $R$ is at the peak of the curve, and there is no phase noise in the system. Under the influence of the memristor regulator, the neuron system generates sparse pulse discharge. When the noise intensity $D=0.75$, the coherence resonance coefficient $R$ is at the valley of the curve. At this time, the system presents a cluster discharge mode of period 1 , and the discharge regularity of the system is the highest and the system is more stable. When the noise intensity $D=3.5$, the larger noise intensity value after the valley is selected as the comparison. And it is found that the time interval of the system discharge peak is irregular, and the discharge order becomes worse. In conclusion, by discretely selecting the period value $T$ of phase noise, it is verified that the neuron system exhibits coherence resonance behavior with the gradual increase of noise intensity $D$.

\section{Conclusion}

In this paper, the FHN neuron system induced by electromagnetic field driven by phase noise is used as the analysis model, with the memristor parameters determined, and the amplitude, period and noise intensity of phase noise as the parameters. The dynamic analysis of coherence resonance behavior of FHN neuron system under two-parameter variation is made from three perspectives: amplitude and period of phase noise, amplitude and noise intensity of phase noise as well as noise intensity and period of phase noise. When the amplitude and period of the phase noise are changed as two-parameter, the FHN neuron system shows coherence multi-resonance behavior. And when the amplitude and period parameters are small, the coherence resonance behavior of the neuron system is the most obvious; when the amplitude and period increase simultaneously, the coherence resonance coefficient appears as a ripple-like rise state. And the system evolves with the increasing number of periodic cluster discharge periods at the valley. When the amplitude and intensity of phase noise are changed as two-parameter, the FHN neuron system exhibits coherence mono-resonance and coherence bi-resonance behavior. It is found that when the system is analyzed with the amplitude as an independent variable within the measurement range of the noise intensity, the change of the system shows the same change tendency. That is, with any value within the range of the noise intensity and the change of amplitude, the system can show the coherence resonance behavior. The FHN neuron system exhibits coherence mono-resonance and coherence multi-resonance behavior when the phase noise intensity and period are changed as two-parameter, and the neuron system is dominated by a large range of coherence resonance. When the phase noise intensity and period are small, several coherence resonances of narrow areas appear. There is noise similar to phase noise in the nervous system, and there is electromagnetic induction in the nervous system. Therefore, it is of great significance to study the coherent resonance behavior of neuron system under the influence of phase noise by using memristor as the electromagnetic induced current, which is generated by nerve cells under the influence of electromagnetic field and plays a regulation role on the membrane potential. The study also has important reference value for analyzing the stochastic dynamic behavior of neuron system.

\section{Data Availability}

https://github.com/0619679/Coherence-Resonance-Behavior-of-FHN-Neurons-Induced-by-Electromagnetic-FieldDriven-by-Phase-Noise

\section{Conflicts of Interest}

The authors declare that they have no conflict of interest.

\section{Acknowledgments}

This work was supported by the National Natural Science Foundation of China (No. 61966022).

\section{References}

[1] L. Glass, "Synchronization and rhythmic processes in physiology," Nature, vol. 410, no. 6825, pp. 277-284, 2001.

[2] K. Wu, T. Wang, C. Wang, T. Du, and H. Lu, "Study on electrical synapse coupling synchronization of HindmarshRose neurons under Gaussian white noise," Neural Computing \& Applications, vol. 30, no. 2, pp. 551-561, 2018.

[3] Y. Yang, Y. H. Cui, K. N. Sang et al., "Ketamine blocks bursting in the lateral habenula to rapidly relieve depression," Nature, vol. 554, no. 1, pp. 317-322, 2018.

[4] A. Mondal, R. K. Upadhyay, J. Ma, B. K. Yadav, S. K. Sharma, and A. Mondal, "Bifurcation analysis and diverse firing activities of a modified excitable neuron model," Cognitive neurodynamics, vol. 13, no. 4, pp. 393-407, 2019.

[5] Y. J. Shen, Y. F. Guo, and B. Xi, "Steady state characteristics in FHN neural system driven by correlated non-Gaussian noise and Gaussian noise," Acta Physica Sinica, vol. 65, no. 12, pp. 51-57, 2016.

[6] T. Li, K. Wu, M. Yan, Z. Liu, and H. Zheng, "Stochastic dynamic behavior of FitzHugh-Nagumo neurons stimulated by white noise," International Journal of Modern Physics B, vol. 35, no. 10, Article ID 2150137, 2021.

[7] Y. Xu, M. Liu, Z. Zhu, and J. Ma, "Dynamics and coherence resonance in a thermosensitive neuron driven by photocurrent," Chinese Physics B, vol. 29, no. 9, Article ID 098704, 2020.

[8] X. L. Li and L. J. Ning, "Stochastic resonance in FizHughNagumo model driven by multiplicative signal and nonGaussian noise," Indian Journal of Physics, vol. 89, no. 2, pp. 189-194, 2015.

[9] C. Xu and Y. M. Kang, "Mean response time of FitzHughNagumo model in the presence of non-Gaussian noise and a periodic signal," Acta Physica Sinica, vol. 60, no. 10, pp. 742-749, 2011.

[10] Y. L. Chen, L. C. Yu, and Y. Chen, "Reliability of weak signals detection in neurons with noise," Science China (Technological Sciences), vol. 59, no. 3, pp. 411-419, 2016.

[11] X. J. Dong and A. J. Yan, "The relationship between stochastic resonance and coherence resonance in a bi-stable system," Acta Physica Sinica, vol. 62, no. 7, pp. 56-62, 2013.

[12] A. Vishwamittar, P. Batra, and R. Chopra, "Stochastic resonance in two coupled fractional oscillators with potential and 
coupling parameters subjected to quadratic asymmetric dichotomous noise," Physica A: Statal Mechanics and its Applications, vol. 561, no. 1, Article ID 125148, 2020.

[13] Y. G. Leng and T. Y. Wang, "Numerical research of twice sampling stochastic resonance for the detection of a weak signal submerged in a heavy Noise," Acta Physica Sinica, vol. 52, no. 10, pp. 2432-2437, 2003.

[14] X. M. Liang, L. Zhao, and Z. H. Liu, "Phase-noise-induced resonance in a single neuronal system," Physical Review, vol. 84, no. 3, Article ID 031916, 2011.

[15] X.-S. Kang, X.-M. Liang, and H.-P. Lü, "Enhanced response to subthreshold signals by phase noise in a hodgkin-huxley neuron," Chinese Physics Letters, vol. 30, no. 1, Article ID 018701, 2013.

[16] C. E. Carr and M. A. Friedman, "Evolution of time coding systems,” Neural Computation, vol. 11, no. 1, pp. 1-20, 1999.

[17] Y. Zhang, G. Hu, and L. Gammaitoni, "Signal transmission in one-way coupled bistable systems: noise effect," Physical Review, vol. 58, no. 3, pp. 2952-2956, 1998.

[18] G. Hu, T. Ditzinger, C. Z. Ning, and H. Haken, "Stochastic resonance without external periodic force," Physical Review Letters, vol. 71, no. 6, pp. 807-810, 1993.

[19] A. Neiman, "Coherence resonance," Scholarpedia, vol. 2, no. 11, 2007.

[20] S.-G. Lee, A. Neiman, and S. Kim, "Coherence resonance in a Hodgkin-Huxley neuron,” Physical Review, vol. 57, no. 3, pp. 3292-3297, 1998.

[21] T. Ditzinger, C. Z. Ning, and G. Hu, "Resonancelike responses of autonomous nonlinear systems to white noise," Physical Review, vol. 50, no. 5, pp. 3508-3516, 1994.

[22] X. Q. Liu and X. L. Yang, "Coherence resonance in a modified FHN neuron with autapse and phase noise," International Journal of Modern Physics B, vol. 32, no. 30, 2018.

[23] X. L. Ding, B. Jia, Y. Y. Li, and H. G. Gu, "Enhancement of coherence resonance induced by inhibitory autapse in Hodgkin-Huxley model," International Journal of Modern Physics B, vol. 35, no. 7, Article ID 2150110, 2021.

[24] Y. Jia, H. Gu, Y. Li, and X. Ding, "Inhibitory autapses enhance coherence resonance of a neuronal network," Communications in Nonlinear Science and Numerical Simulation, vol. 95, Article ID 105643, 2021.

[25] L. Ryashko and E. Slepukhina, "Noise-induced toroidal excitability in neuron model," Communications in Nonlinear Science and Numerical Simulation, vol. 82, Article ID 105071, 2020.

[26] M. S. Wang, W. X. Huang, and Z. F. Cui, "Coherence biresonance in a two-dimensional neural map," Acta Physica Sinica, vol. 59, no. 7, pp. 4485-4489, 2010.

[27] Z. H. Liu, Y. R. Zhou, A. Y. Zhang, and X. F. Pang, "Coherence resonance in a nonlinear neuronal models driven by correlated colored noise," Acta Physica Sinica, vol. 59, no. 2, pp. 699-704, 2010.

[28] Y. Jia and H. Gu, "Phase noise-induced double coherence resonances in a neuronal model," International Journal of Modern Physics B, vol. 29, no. 20, Article ID 1550142, 2015.

[29] Y. Jia and H. Gu, "Transition from double coherence resonances to single coherence resonance in a neuronal network with phase noise," Chaos: An Interdisciplinary Journal of Nonlinear Science, vol. 25, no. 12, Article ID 123124, 2015.

[30] A. S. Pikovsky and J. Kurths, "Coherence resonance in a noisedriven excitable system," Physical Review Letters, vol. 78, no. 5, pp. 775-778, 1997.

[31] J. Casado, "Noise-induced coherence in an excitable system," Physics Letters A, vol. 235, no. 5, pp. 489-492, 1997.
[32] L. Chua, "Memristor-The missing circuit element," IEEE Transactions on Circuit Theory, vol. 18, no. 1, pp. 507-519, 1971.

[33] D. B. Strukov, G. S. Snider, D. R. Stewart, and R. S. Williams, "The missing memristor found," Nature, vol. 453, no. 1, pp. 80-83, 2008.

[34] C. Li, W. J.-C. Thio, H. H.-C. Iu, and T. Lu, "A memristive chaotic oscillator with increasing amplitude and frequency," IEEE Access, vol. 6, no. 1, Article ID 12945, 2018.

[35] C. Li, F. Min, and C. Li, "Multiple coexisting attractors of the serial-parallel memristor-based chaotic system and its adaptive generalized synchronization," Nonlinear Dynamics, vol. 94, no. 4, pp. 2785-2806, 2018.

[36] B. Bao, G. Shi, J. Xu, Z. Liu, and S. Pan, "Dynamics analysis of chaotic circuit with two memristors," Science China Technological Sciences, vol. 54, no. 8, pp. 2180-2187, 2011.

[37] Q. Xu, Y. Lin, B. Bao, and M. Chen, "Multiple attractors in a non-ideal active voltage-controlled memristor based Chua's circuit," Chaos, Solitons \& Fractals, vol. 83, pp. 186-200, 2016.

[38] G. Zhang, J. Ma, A. Alsaedi, B. Ahmad, and F. Alzahrani, "Dynamical behavior and application in Josephson Junction coupled by memristor," Applied Mathematics and Computation, vol. 321, pp. 290-299, 2018.

[39] J. Ma, L. Mi, P. Zhou, Y. Xu, and T. Hayat, "Phase synchronization between two neurons induced by coupling of electromagnetic field," Applied Mathematics and Computation, vol. 307, pp. 321-328, 2017.

[40] M. Ge, L. Lu, Y. Xu, R. Mamatimin, Q. Pei, and Y. Jia, "Vibrational mono-/bi-resonance and wave propagation in FitzHugh-Nagumo neural systems under electromagnetic induction," Chaos, Solitons \& Fractals, vol. 133, Article ID 109645, 2020.

[41] R. Fitzhugh, "Impulses and physiological states in theoretical models of nerve membrane," Biophysical Journal, vol. 1, no. 6, pp. 445-466, 1961.

[42] J. Nagumo, S. Arimoto, and S. Yoshizawa, "An active pulse transmission line simulating nerve axon," Proceedings of the Ire, vol. 50, no. 10, pp. 2061-2070, 1962.

[43] Y. Liu and Y. Xie, "Dynamical characteristics of the fractionalorder FitzHugh-Nagumo model neuron and its synchronization," Acta Physica Sinica, vol. 59, no. 3, pp. 2147-2155, 2010.

[44] Y. T. Yang and S. Q. Liu, "Firing patterns and bifurcation analysis of FHN-ML electrically coupled neurons," Chinese Journal of Medical Physics, vol. 34, no. 3, pp. 306-313, 2017. 